\title{
Real time observation of chaperone-modulated talin mechanics under single molecule resolution
}

\author{
Souradeep Banerjee ${ }^{\dagger}$, Deep Chaudhuri ${ }^{\dagger}$, Soham Chakraborty ${ }^{\dagger}$, Shubhasis Haldar ${ }^{*}$ \\ Department of Biological Sciences, Ashoka University, Sonepat, Haryana 131029 \\ $\dagger$ contributed equally to this work \\ * to whom correspondence may be addressed. Email- shubhasis.halder@ ashoka.edu.in
}




\begin{abstract}
Recent single molecule studies have recognized talin as a mechanosensitive hub in focal adhesion, where its function is strongly regulated by mechanical force. For instance, at low force (less than $5 \mathrm{pN}$ ), folded talin binds RIAM for integrin activation; whereas at high force (more than $5 \mathrm{pN}$ ), it unfolds to activate vinculin binding for focal adhesion stabilization. Being a cytoplasmic large protein, talin must interact with various chaperones, however the role of chaperones on talin mechanics is unknown.

To address this question, we investigated the force response of a mechanically stable talin domain, with a set of well-known holdase and foldase chaperones, using a single molecule magnetic tweezers technology. Our findings demonstrate a novel mechanical role of chaperones. We found holdase chaperones reduce the mechanical stability of the protein to $\sim 6 \mathrm{pN}$, while the foldase chaperone increases it up to $\sim 15 \mathrm{pN}$. The alteration in mechanical stability ascribes to the underlying molecular mechanism where the chaperones directly reshape the energy landscape of talin. For example, unfoldase chaperone (DnaK) decreases the unfolding barrier height from 26.8 to $21.69 \mathrm{k}_{\mathrm{B}} \mathrm{T}$ and increases the refolding barrier from 3.49 to $11.31 \mathrm{k}_{\mathrm{B}} \mathrm{T}$. In contrast, foldase chaperone (DsbA) increases the unfolding barrier to $33.46 \mathrm{k}_{\mathrm{B}} \mathrm{T}$ and decreases the refolding barrier to $0.44 \mathrm{k}_{\mathrm{B}} \mathrm{T}$. The quantitative mapping of the chaperone-induced free energy landscape of talin directly shows that chaperones could perturb the focal adhesion dynamics, which in turn can influence downstream signaling cascades in diverse cellular processes.
\end{abstract}




\section{Introduction:}

Cells interpret and respond to complex mechanical environment through mechanotransduction- a comprehensive focal adhesion (FA) mediated response which helps to transduce mechanical cues into biochemical signals, supporting diverse biological processes such as, cell development, migration, and proliferation. ${ }^{1}$ These mechanical communications, through the large adhesion complex, are tightly regulated by the physical linkages of mechanosensing proteins. Talin is a key mechanosensitive scaffold protein which connects transmembrane integrin to actin cytoskeleton, and mechanically tunes diverse interactions by force transmission. ${ }^{2-5}$ Talin rod segment (R1-R13) exhibits conformational changes and hierarchical unfolding of each rod domains under increasing mechanical tension. ${ }^{6-8}$ Among them, R3 is the most unstable domain that not only unfolds at $\sim 5 \mathrm{pN}$ but also exhibits equilibrium folding-unfolding transitions on a sub-second timescale by a single actomyosin contraction. ${ }^{2,8,9}$ This force-dependent folding dynamics allow them to tune their interactome profile: such as, Rap1-interacting adapter molecule (RIAM) binds to folded R3 domain and protects from mechanical unfolding, whereas force above $5 \mathrm{pN}$ results in stepwise unfolding of the domain with subsequent vinculin binding. This mutual exclusive interaction allows talin to acts as a mechanochemical switch, shifting from an initial integrin activator to the active mediator of adhesion maturation and stabilization. ${ }^{2,10}$ Interestingly molecular chaperones, involved in various folding related process starting from protein translation to degradation, could play a pivotal role in FA stabilization by colocalizing with different cytoskeletal and adhesion proteins. ${ }^{11-13,13-17}$ Being a critical mechanosensitive protein in the focal adhesion, talin must interact with different cellular chaperones; however, there is no direct evidence on how chaperones mechanically influence its interaction profiles during the adhesion maturation process.

To address this question, single molecule real-time microfluidics magnetic tweezers (RTMMT) has been used, which executes both force ramp and force clamp technologies. ${ }^{18}$ The force ramp technology, by increasing or decreasing the force at a constant rate, probes unfolding and refolding events of a protein; while applying the force clamp technology, a constant force can be applied to the protein, allowing to detect their thermodynamics and kinetic properties under equilibrium condition. Owing to the advantage of wide force regime of $0-120 \mathrm{pN}$ with sub-pN resolution, we were able to observe chaperone interaction both at folded and unfolded state, independently. Additionally, the associated microfluidic chamber allows us to introduce chaperones, individually or in combination, to probe their effect on folding dynamics of a single protein in real time. Finally, implementing this methodology, the force can be specifically applied on the client protein while the chaperones remain unperturbed.

Here, we systematically investigated the chaperone effects on the mechanical stability of talin R3 domain, with a set of model chaperones: holdase, foldase and mechanically neutral. While the holdases hinder the folding process by stabilizing the unfolded state, foldase chaperones accelerate the folding process under force. However, the mechanically neutral chaperones do not exhibit any activity, restoring only the intrinsic folding ability in client protein as 
comparable to the absence of any chaperones. We observed oxidoreductase enzyme DsbA as foldase, increases the mechanical stability of R3-IVVI domain by increasing their unfolding force from 10 to $15 \mathrm{pN}$, whereas DnaJ and DnaK as unfoldase, reduce the mechanical stability by reducing the force to $6 \mathrm{pN}$. Furthermore, our results showed chaperones could reshape the free energy landscape of substrate proteins by changing the height of their free energy barrier without significantly affecting the transition state distance, which resolves the quantitative description of the chaperone modulated free energy landscape. Overall, our study proposed how chaperone affects the mechanical stability of focal adhesion protein- talin, which could also regulate cellular mechanochemical signalling.

\section{Results:}

\section{Single molecule folding dynamics of Talin R3-IVVI domain:}

The talin R3 domain is the mechanically weakest, due to the presence of four threonine residues at its hydrophobic core, and unfolds earlier than any other rod domains of talin, exhibiting the equilibrium folding dynamics at $\sim 5 \mathrm{pN}$, a lower limiting force range of the magnetic tweezers. ${ }^{8,19}$ As the holdase chaperones are capable of stabilizing the unfolded state, they are expected to shift the folding dynamics below $4 \mathrm{pN}$ and thus, a mechanically stable version of R3 domain is required. Therefore, by substituting these four threonine residues with larger hydrophobic residues containing amino acids- isoleucine and valine at 809,833 , 867 and 901 position, a mechanically stable R3-IVVI mutant domain has been used for this study. Both the wild type R3 and R3-IVVI domains have been extensively studied and observed to share similar function. However, R3-IVVI exhibits amplified mechanical signature during the experiment. For example, both of them binds vinculin at unfolded state but wild type R3 domain unfolds at $5 \mathrm{pN}$, whereas R3-IVVI mutant unfolds at 9 pN. ${ }^{10,20,21}$

The real time folding-unfolding dynamics of R3-IVVI domain, at single molecular resolution, has been observed using RT-MMT technology. This technology allowed the application of sub-pN level of force to understand the folding dynamics of the protein at equilibrium. This single-molecule force spectroscopy technology provides two ways for experimentation- force ramp and force clamp techniques. The talin construct is covalently attached to glass surface, using the HaloTag covalent chemistry and the C-terminal Avi-tag was biotinylated to bind the paramagnetic beads through biotin-streptavidin chemistry (Fig. 1A). Force is applied on the paramagnetic bead with a pair of permanent magnets, attached to a voice coil actuator, which controls the amount of force as an inverse function of the distance between permanent magnet and the paramagnetic beads. ${ }^{22}$

The force clamp technology allowed to provide a distinct force on the substrate and enabled us to observe its oscillation between two conformations: folded and unfolded states. Fig. 1B shows three representative trajectories of talin R3-IVVI folding dynamics, and the population of both the folded and unfolded states, at three distinct forces. The folding probability was deduced by analysing several of these folding trajectories and dividing the relative population of folded state by the total duration of the observed dynamics as described previously. ${ }^{20,21}$ At 
$10 \mathrm{pN}$, the talin R3-IVVI domain displays almost equal proportion of folded and unfolded conformation with an extension change of $\sim 20 \mathrm{~nm}$; while at $8.5 \mathrm{pN}$ and $11.5 \mathrm{pN}$, the domain mostly occupies folded and unfolded population with a folding probability of 0.87 and 0.1 , respectively. Using the force ramp technology, the mechanical stability of R3-IVVI was monitored where the force was increased from 4 to $35 \mathrm{pN}$ and decreased in the consecutive cycle at a constant rate of $3.1 \mathrm{pN} / \mathrm{sec}$. It was observed that R3-IVVI unfolds at $10.7 \pm 0.21 \mathrm{pN}$, whereas refolds at $9.9 \pm 0.23 \mathrm{pN}$ (Fig. 1C). By considering tens of such successive force ramp experiments on each molecule, the quantified amount of unfolding and refolding force of Talin R3-IVVI was significantly concluded.

\section{Effect of chaperones on the unfolding force of the Talin R3-IVVI domain:}

We systematically investigated the unfolding and refolding force of R3-IVVI domain using the force ramp protocol, in the presence and absence of different chaperones. In unfolding ramp experiment, we increased the force from 4 to $35 \mathrm{pN}$ at a constant loading rate of 3.1 $\mathrm{pN} / \mathrm{sec}$ to monitor unfolding event against force.

In Fig. 2A, we observed the decrease in unfolding force of R3-IVVI with increasing concentrations of DnaJ, a prototypical holdase chaperone, ${ }^{23}$ in comparison to the control. In case of control or absence of any chaperones in the solution, the unfolding force of the substrate is $\sim 11 \mathrm{pN}$, whereas in the presence of $1 \mu \mathrm{M}$ DnaJ, the unfolding force is reduced to $\sim 7 \mathrm{pN}$ (Fig 2A). Similarly, we performed this experiment in the presence of variable concentration of DnaK individually (Fig. 2B) and in the presence of DnaJ, DnaK, and ATP together (Fig. 2C). In the both cases, the unfolding rate of R3-IVVI decreased as compared to the absence of any chaperones. In the presence of DnaK and DnaJ together, the unfolding force of talin R3-IVVI is increased to $9.4 \pm 0.7 \mathrm{pN}$ than DnaJ or DnaK alone, but still working as a holdase in comparison to the control. However, in presence of DnaKJE (consisting DnaK, DnaJ, GrpE and $10 \mathrm{mM}$ ATP), we observed the unfolding event of talin at $10.8 \pm 0.7$ pN (Fig. 2C). Remarkably, in the presence of oxidized DsbA, a known force dependent foldase chaperone, ${ }^{24}$ the mechanical stability of the substrate to $\sim 15 \mathrm{pN}$ (Fig. 2D).

\section{Effect of chaperone on the refolding force of talin R3-IVVI:}

The refolding force was statistically characterized for the talin R3-IVVI domain (control), to be $9.9 \pm 0.23 \mathrm{pN}$, using successive ramp experiments, by decreasing the force from 35 to $4 \mathrm{pN}$ at a rate of $3.1 \mathrm{pN} / \mathrm{sec}$, as shown in fig. 1C. We observed the effect of holdase (DnaK and DnaJ) and foldase (DnaKJE and DsbA) chaperones on the refolding force of talin R3-IVVI. Gradual increment in the concentration of DnaJ from 0.5 to $3 \mu \mathrm{M}$ declined the refolding force of talin to $\sim 7 \mathrm{pN}$ (Fig. 3A). Whereas, in presence of $3 \mu \mathrm{M}$ DnaK, the refolding rate decreased to almost a similar force range as caused by DnaJ (Fig. 3B). On addition, in presence of DnaJ with DnaK and $5 \mathrm{mM}$ ATP, the refolding rate was reduced to $7.56 \pm 0.7 \mathrm{pN}$ in compared to control. On addition of GrpE to this solution, we observed the refolding rate increased to $9.78 \pm 0.6 \mathrm{pN}$ (Fig 3C). However, with the foldase chaperone, oxidised DsbA, was provided at 
$30 \mu \mathrm{M}$ and $60 \mu \mathrm{M}$, it showed a concentration dependent increment of refolding force to $\sim 11$ $\mathrm{pN}$ and $13 \mathrm{pN}$ respectively (Fig. 3D).

\section{Chaperones shifts the folding probability of the substrate under force:}

Using the force clamp method, we determined the folding probability (FP) by dwell time distribution, from several equilibrium trajectories at different forces. Folding probability is the relative population of the folded state within the observed duration of equilibrium phase (Fig. 1B) and is strongly dependent on applied force. ${ }^{20,21}$ We calculated the folding dynamics at different forces, in the presence and absence of chaperones, and calculated the half-point force in each occasion where the folded and unfolded populations reside almost equally. We observed that the half-point force of R3-IVVI is $9.76 \mathrm{pN}$, however, chaperones have the ability to shift this half-point force either towards higher or lower force. We observed that in the presence of $1 \mu \mathrm{M}$ DnaJ and $3 \mu \mathrm{M}$ DnaK, these holdase chaperones shift the half-point force from 9.76 to $\sim 8 \mathrm{pN}$, and $\sim 6 \mathrm{pN}$, respectively (Fig. 4A and 4B). However, cytoplasmic chaperone DnaKJE complex restores the half-point force to $9.8 \mathrm{pN}$ of R3-IVVI, in comparison to DnaJ and DnaK together (8.7 pN) (Fig. 4C). Notably, $60 \mu \mathrm{M}$ of oxidised DsbA has the ability to upshift the equilibrium force to $14.7 \mathrm{pN}$ from $9.76 \mathrm{pN}$ (Fig. 4D).

\section{Unfolding and refolding kinetics of Talin R3 observed under force in presence and absence of chaperones:}

By using dwell time analysis of several equilibrium trajectories at different forces, in the absence and presence of chaperones, the unfolding and refolding rate of talin R3-IVVI were calculated as described previously. ${ }^{20}$ The rate was derived from the inverse of average of the folded and unfolded dwell time, at particular force, as determined from exponential fit to the dwell time distribution (eq. 1 and 2). As talin R3-IVVI domain exhibits folding-unfolding transitions within a small force regime of $2 \mathrm{pN}$, the data could be explained by Bell model. ${ }^{20}$ By fitting these data in a Bell model, the $\ln$ of force dependent rates were obtained.

$$
\begin{aligned}
& \ln k_{U}=\left[\ln A-\left(\frac{\Delta G_{U}}{k_{B} T}\right)\right]+\left(\frac{F \Delta x_{U}}{k_{B} T}\right) \\
& \ln k_{F}=\left[\ln A-\left(\frac{\Delta G_{F}}{k_{B} T}\right)\right]-\left(\frac{F \Delta x_{F}}{k_{B} T}\right)
\end{aligned}
$$

These data showed that gradual increase in force, is directly proportional to unfolding rate and inversely to refolding rate (Fig. 5A). The intersection of the unfolding and refolding rate is called the half-point force where the substrate shows an almost equal proportion of folded and unfolded states. However, chaperone interaction shifts the half-point force of R3-IVVI to a different force range. Such as, holdase like DnaJ or DnaK shift the half-point force of R3IVVI from $10 \mathrm{pN}$ to $8 \mathrm{pN}$ and $7 \mathrm{pN}$, respectively (Fig. 5B and 5C). Whereas mechanical foldase chaperone, like DsbA, increased the half-point force to15 pN (Fig. 5D). 


\section{Discussion:}

Force response of talin domains has been well characterized using force spectroscopy and stochastic kinetic stimulation where R3 domain has been observed to be the weakest domain, ${ }^{8}$ exhibiting folding-unfolding dynamics at $\sim 5 \mathrm{pN}$. This mechanical instability is originated due to unzipping force geometry and a unique threonine belt at hydrophobic core. ${ }^{10}$ Though R3 domain unfolds at $<5 \mathrm{pN}$ force, an energy cost of above $10 \mathrm{k}_{\mathrm{B}} \mathrm{T}$ is required for the unfolding of the domain due to the large unfolding extension of $\sim 20 \mathrm{~nm} .{ }^{25}$ Interestingly, chaperones could modulate the mechanical response of the talin domains, however, the molecular mechanism of these interactions under force still remains elusive. RT-MMT technology, using fore-clamp protocol, allows us to monitor the chaperone activity on the mechanical stability of R3-IVVI domain. Although chaperones are not able to change the transition state distance significantly, they reshape the free energy landscape by changing the height of the free energy barrier with respect to the folded/unfolded state. The unfoldase chaperone DnaJ acts by two specific ways: it stabilizes the unfolded state by decreasing the unfolding free energy barrier $\left(\Delta \mathrm{G}_{\mathrm{U}}^{\dagger}\right)$ from $26.8 \pm 0.83$ to $21.69 \pm 0.3 \mathrm{k}_{\mathrm{B}} \mathrm{T}$, and increasing the refolding energy barrier $\left(\Delta \mathrm{G}_{\mathrm{F}}^{\dagger}\right)$ from $3.49 \pm 0.47$ to $7.27 \pm 0.43 \mathrm{k}_{\mathrm{B}} \mathrm{T}$. This tilts the energy landscape of R3-IVVI towards the unfolded state (Table 1 and 2), which can also be explained from the decreased folding probability (Fig. 4A and B). However, DnaKJE complex as a well-known foldase, ${ }^{23,26-30}$ has only been observed to restore the intrinsic folding ability of R3-IVVI domain as comparable to the absence of any chaperones. Interestingly, DsbA as a foldase chaperone shifts the free energy landscape towards the folded state by increasing $\Delta \mathrm{G}_{\mathrm{U}}^{\dagger}$ to $33.46 \pm 0.49 \mathrm{k}_{\mathrm{B}} \mathrm{T}$ and decreasing $\Delta \mathrm{G}_{\mathrm{F}}^{\dagger}$ to $0.44 \pm 0.08 \mathrm{k}_{\mathrm{B}} \mathrm{T}$ (Table 1 and 2) which in turn increases the folding probability (Fig. 4D). This chaperone-mediated reshaping of the free energy landscape apparently affects the mechanical stability of the R3 domain, allowing it to perform as a regulatory mechanical band-pass filter within a narrow force frequency, to convert complex mechanical signals. Therefore, from a broader viewpoint, this could largely impact the force transduction into biochemical signalling by changing the force-dependent folding dynamics.

Mechanotransduction events largely relies on robust and precise mechanical response of molecular force springs which decode the mechanical signals by both the force magnitude and their temporal resolution. Talin rod domains have exquisite ability to interpret these signals by force driven structural dynamicity and concurrent modulation of complex interactome profiles. These rod domains have graded mechanical stability and unfolds sequentially at increasing tension to control the adhesion dynamics. ${ }^{8,19}$ Among them, R3 domain acts as initial mechanosensor, initiating talin activation and subsequent signalling pathways. At low force $(<5 \mathrm{pN}), \mathrm{R} 3$ domain engages to RIAM via folded state, and becomes protected against the mechanical stretching. The tension-induced unfolding at $>5 \mathrm{pN}$ and concurrent RIAM dissociation is an essential criterion for vinculin binding to cryptic binding helices (vinculin binding site, VBS). Recently, pressure dependent chemical shift has also observed that R3 domain is thermodynamically tuned for interacting with either RIAM or 
vinculin which undergoes through a stepwise structural transition. ${ }^{2}$ This mutual exclusive interaction of RIAM and vinculin to R3 finely calibrates downstream signalling cascades, reflecting the changes in cell morphology. ${ }^{31}$ RIAM recruitment to talin-integrin mechanical linkage results in filopodial protrusion in nascent adhesion that eventually replaced by vinculin in mature adhesion. ${ }^{9}$ The adhesion reinforcement results from the actomyosin contractility which regulate the FA dynamics by tension-dependent binding constant of antagonistic partners. For example, RIAM, DLC1 binding to folded domains, can stabilize talin by increasing the mechanical force threshold for vinculin binding. R3 stabilization has been reported to affect fibroblast matrix rigidity sensing and YAP (yes associated protein) signalling, while destabilized R3 exhibits conformational dynamics and are more prone to tension-induced unfolding. ${ }^{32}$ This unfolding at relatively low force, and subsequent vinculin binding plausibly activate downstream signalling pathways that decrease traction force generation. In mature adhesion, actomyosin contractility perturbs RIAM binding, abrogating its negative effect on RhoA signalling which enhances the tension at growing adhesion. However, as the adhesion matures and engage more proteins to the complex, the resultant force is decreased on individual talin molecules due to multiple parallel linkage. This force reduction on each mechanical linkage, as force-bearing springs, has been observed by FRET tension sensor and has been reported to be decreased in mature focal adhesion complex than nascent adhesion at cell periphery. ${ }^{33,34}$ This means R3 domain again switch to RIAM binding state and reduces the force transmission through successive domains and thus, regulates focal adhesion dynamics (Fig. 7). However, focal adhesion has also been thought to be controlled by active participation of molecular chaperones with different adhesion proteins, where they could modulate the folding-unfolding transitions of substrate proteins and thus, their interaction with other partners. In this sense, it remains to be investigated whether transient interaction of chaperones could also play a pivotal role in mechanotransduction where force triggers conformational changes of mechanosensing proteins, and thereby, regulating downstream signalling pathways.

Interestingly, there is emerging evidence to suggest that molecular chaperones could accelerate the folding-unfolding transition of cytoskeletal proteins such as actin and tubulin. ${ }^{13}$ Studies have shown that chaperones like $\alpha \beta$ crystallin or calreticulin has played crucial role in deciding mechanical properties of cell, like resilience of cell adhesion, cell spreading, by regulating expression of key focal adhesion proteins. ${ }^{13,14}$ Small heat shock proteins have also been observed to regulate chronic biomechanical stress in heart tissue by regulating dynamics of key FA protein like actin and filamin C. ${ }^{17}$ Additionally, tension-induced ubiquitylation of filamin A promotes chaperone-assisted autophagy which in turn dictate the role of chaperones in focal adhesion assembly. ${ }^{35,36}$ However, the underlying molecular mechanism of these chaperone interactions, during mechanotransduction, has not been reported yet. Additionally, chaperones are known to assists folding by preventing misfolding, stabilizing unfolded state or acting as dis-aggregate, their mechanical role differs slightly than these observed roles. ${ }^{11,23,26-28}$ Such as, known cytoplasmic foldase DnaKJE complex, which has the ability to fold its substrate, is only able to restore the mechanical stability in talin R3 under force. However, cytoplasmic holdase like DnaK or DnaJ, acts by stabilizing the unfolded state of the substrate both in the presence and absence of force. Interestingly, tunnel 
associated chaperones possess an ability to increase the mechanical stability of their substrates. Such as oxidoreductase DsbA, a well-known model mechanical foldase, has the ability to increase the folding rate and mechanical stability of R3. ${ }^{24,37,38}$ Hence, our results demonstrate that chaperones could modulate the mechanical stability of the FA protein talin, which are characterized by the change in the free energy barrier. Additionally, we propose this change in mechanical stability of R3 domain, can significantly affect binding kinetics with RIAM and vinculin: foldase chaperones facilitate the RIAM binding by shifting the energy barrier towards folded state and slows the stepwise unfolding of the domain, while unfoldase chaperones promote vinculin binding by decreasing the unfolding free energy barrier. This could convert the switch response of R3 domain into a domain-locked response, disrupting the natural downstream signalling pathway to control the diverse cellular processes.

\section{Materials and Methods:}

\section{Expression and purification of talin:}

We used the mechanically stable talin R3-IVVI domain as a model protein, as previously reported in force spectroscopic studies. ${ }^{20,21}$ For purification, the protein construct was transformed into the Escherichia coli BL21 (DE3 Invitrogen) competent cells. Cells were grown in luria broth at $37^{\circ} \mathrm{C}$ with carbenicillin, till the O.D. becomes $0.6-0.8$ at $600 \mathrm{~nm}$. The cultures were then induced with $1 \mathrm{mM}$ Isopropyl $\beta$-D-thiogalactopyranoside (IPTG, SigmaAldrich) overnight at $25^{\circ} \mathrm{C}$. The cells were pelleted and re-suspended in $50 \mathrm{mM}$ sodium phosphate buffer $\mathrm{pH} 7.4$, containing $300 \mathrm{mM} \mathrm{NaCl}$ and $10 \%$ glycerol. $10 \mu \mathrm{M}$ Phenylmethylsulfonyl (PMSF) was used as protease inhibitor followed by lysozyme for membrane lysis. After incubating the solution for 20 minutes at $4^{\circ} \mathrm{C}$, dissolved pellet was treated with Triton-X 100 (Sigma Aldrich), DNase, RNase (Invitrogen) and $10 \mathrm{mM} \mathrm{MgCl}$ and kept at $4^{\circ} \mathrm{C}$ in rocking platform. The cells were disrupted in French press and cell lysate was centrifuged at $11000 \mathrm{rpm}$ for $1 \mathrm{~h}$. The protein was purified from the lysate using $\mathrm{Ni}^{2+}$ NTA column of $\square$ KTA Pure (GE healthcare). For in vitro biotinylation of the polyprotein, Avidity biotinylation kit was used and the biotinylated polypeptide was purified by Superdex200 increase 10/300 GL gel filtration column in presence of Na-P buffer with $150 \mathrm{mM}$ $\mathrm{NaCl}^{38}$

\section{Expression and purification of Chaperones (DnaJ, DnaK, GrpE and DsbA):}

For purification of DnaJ and GrpE protein purification, these constructs were transformed in Escherichia Coli (DE3) cells and grown in luria broth media at $37^{\circ} \mathrm{C}$ with respective antibiotics (carbenicillin and ampicillin, 50 $\mathrm{g} / \mathrm{ml}$ ). The cells were induced overnight with Isopropyl $\beta$-D-thiogalactopyranoside (IPTG, Sigma-Aldrich) at $25^{\circ} \mathrm{C}$. Cells were centrifuged and the pellet was re-suspended in $50 \mathrm{mM}$ sodium phosphate buffer, $10 \%$ glycerol and 300 $\mathrm{mM} \mathrm{NaCl}, \mathrm{pH}$ 7.4. The solution was then incubated with PMSF and lysozyme. DNase, RNase, $\mathrm{MgCl}_{2}$ and Triton-X 100 was mixed subsequently at $4^{\circ} \mathrm{C}$. Then the cells were disrupted in French press and the lysate was extracted and purified using $\mathrm{Ni}^{2+}$-NTA affinity 
column of $\square$ KTA Pure (GE healthcare). We used $20 \mathrm{mM}$ imidazole containing buffer as binding buffer and $250 \mathrm{mM}$ imidazole containing sodium phosphate buffer as elution buffer. We purified DnaK from the cell lysate using the $\mathrm{Ni}^{2+}{ }_{-}$NTA column, pre-loaded with Mge1 protein. Then the DnaK protein was eluted with buffer containing $2 \mathrm{mM} \mathrm{ATP}{ }^{39}$ For DsbA, the pellet was dissolved in $50 \mathrm{mM}$ Tris buffer with $1 \mathrm{mM}$ EDTA and $20 \%(\mathrm{w} / \mathrm{v})$ sucrose, $\mathrm{pH}$ 8.0. After 20 minutes of ice incubation the mixture was provided with DNase, RNase and PMSF. Then centrifuged the solution followed by collecting both the supernatant (S-1) and pellet. The pellet was dissolved in $20 \mathrm{mM}$ Tris solution and again centrifuged and the supernatant (S-2) was collected. The purification was done by purifying these two supernatants with $1 \mathrm{M} \mathrm{NaCl}$ containing Tris buffer using Hi-Trap Q-FF anion exchange column of ÄKTA Pure (GE healthcare). To maintain the oxidation activity of DsbA $0.3 \%$ $\mathrm{H}_{2} \mathrm{O}_{2}$ was added to the purified protein for overnight. The protein was further purified by size exclusion chromatography using Superdex-200 increase 10/300 GL gel filtration column in presence of $150 \mathrm{mM} \mathrm{NaCl}{ }^{24}$

\section{Preparation of glass slide and coverslips:}

During RT-MMT experiment, the glass slides were washed with Hellmanex III (1.5\%) solution (Sigma Aldrich) followed by washing with double distilled water. It was then soaked in a mixture containing concentrated hydrochloric acid $(\mathrm{HCl})$ and methanol $\left(\mathrm{CH}_{3} \mathrm{OH}\right)$. The slides were then treated with concentrated sulphuric acid $\left(\mathrm{H}_{2} \mathrm{SO}_{4}\right)$ followed by washing in double distilled water. The glass slides were put into gently boiling water and dried. To activate the glass surface, the glass slides were dissolved in the ethanol solution of 1\% (3Aminopropyl) trymethoxysilane (Sigma Aldrich, 281778) for 15 minutes. Then the glasses were washed with ethanol for removing the unreacted silane and baked them in $65^{\circ} \mathrm{C}$. The coverslips were washed with Hellmanex III (1.5\%) solution for 15 minutes followed by ethanol and dried in the oven for 10 minutes. After sandwiching the glass and coverslips, the chamber was filled with glutaraldehyde (Sigma Aldrich) for an hour. Then the chambers were flashed with reference beads $(2.5-2.9 \mu \mathrm{m}$, Spherotech, AP-25-10) and Halo-Tag (O4) ligand (Promega, P6741). To avoid non-specific interaction the glass chambers were washed with blocking buffer (20 mM Tris-HCl, $150 \mathrm{mM} \mathrm{NaCl}, 2 \mathrm{mM} \mathrm{MgCl}, 0.03 \% \mathrm{NaN}_{3}, 1 \% \mathrm{BSA}$, pH 7.4) for 5 hours at room temperature. ${ }^{18,40}$

\section{Real time multiplexed microfluidic magnetic tweezers (RT-MMT) technology and experiment:}

In RT-MMT technology, the magnetic tweezers set up was built on an inverted microscope using $63 \mathrm{x}$ oil-immersion objective that is attached with a nanofocusing piezo actuator. A linear voice- coil is located above the sample chamber to control the position of the magnet. Images were acquired using ximea camera (MQ013MG-ON). Details information regarding the image processing and techniques are described previously. ${ }^{18}$ RT-MMT experiment was performed with 1-10 nM of protein in a buffer containing $140 \mathrm{mM} \mathrm{NaCl}, 20 \mathrm{mM}$ sodium phosphate and $10 \mathrm{mM}$ ascorbic acid. ${ }^{41,42}$ Ascorbic acid was used as antioxidant for preventing the oxidative damage of the protein. After passing the protein through the chamber, the force is applied on the paramagnetic bead (Dynabeads M-270), attached with Avi-Tagged protein. 
Folding and unfolding dynamics of the protein was observed by applying different force ramp and force clamp protocols. For experiments with DnaKJ and DnaKJE complex, the buffer was supplemented with $10 \mathrm{mM}$ ATP and $10 \mathrm{mM} \mathrm{MgCl}$. The buffer was exchanged after every 30 minutes with fresh ATP to provide sufficient supply of ATP.

\section{Acknowledgement:}

We thank Ashoka University for support and funding. S.H. thanks DBT Ramalingaswami Fellowship and DST SERB Core Research Grant for funding. We thank Prof. Julio Fernandez (Columbia University) for helping us with the magnetic tweezers set up and Dr. Koyeli Mapa of Shiv Nadar University for kindly sharing with us the clones of SecB, DnaK, DnaJ, GrpE proteins. We thank Dr. Rafael Tapia-Rojo (Kings College London) and Dr Edward C. Eckels (Columbia University Medical Center, New York) for the critical analysis and discussion of this work.

\section{Conflict of Interest:}

The authors declare no conflict of interest.

\section{References:}

(1) Chakraborty, S.; Banerjee, S.; Raina, M.; Haldar, S. Force-Directed "Mechanointeractome" of Talin-Integrin. Biochemistry 2019, 58 (47), 4677-4695. https://doi.org/10.1021/acs.biochem.9b00442.

(2) Baxter, N. J.; Zacharchenko, T.; Barsukov, I. L.; Williamson, M. P. PressureDependent Chemical Shifts in the R3 Domain of Talin Show That It Is Thermodynamically Poised for Binding to Either Vinculin or RIAM. Structure 2017, 25 (12), 1856-1866.e2. https://doi.org/10.1016/j.str.2017.10.008.

(3) Calderwood, D. A.; Ginsberg, M. H. Talin Forges the Links between Integrins and Actin. Nature Cell Biology 2003, 5 (8), 694-696. https://doi.org/10.1038/ncb0803-694.

(4) Brakebusch, C.; Fässler, R. The Integrin-Actin Connection, an Eternal Love Affair. The EMBO Journal 2003, 22 (10), 2324-2333. https://doi.org/10.1093/emboj/cdg245.

(5) Nayal, A.; Webb, D. J.; Horwitz, A. F. Talin: An Emerging Focal Point of Adhesion Dynamics. Current Opinion in Cell Biology 2004, 16 (1), 94-98. https://doi.org/10.1016/j.ceb.2003.11.007.

(6) Goult, B. T.; Yan, J.; Schwartz, M. A. Talin as a Mechanosensitive Signaling Hub. J Cell Biol 2018, 217 (11), 3776-3784. https://doi.org/10.1083/jcb.201808061.

(7) Han, S. J.; Azarova, E. V.; Whitewood, A. J.; Bachir, A.; Guttierrez, E.; Groisman, A.; Horwitz, A. R.; Goult, B. T.; Dean, K. M.; Danuser, G. Pre-Complexation of Talin and Vinculin without Tension Is Required for Efficient Nascent Adhesion Maturation. eLife 2021, 10, e66151. https://doi.org/10.7554/eLife.66151.

(8) Yao, M.; Goult, B. T.; Klapholz, B.; Hu, X.; Toseland, C. P.; Guo, Y.; Cong, P.; Sheetz, M. P.; Yan, J. The Mechanical Response of Talin. Nature Communications 2016, 7 (1), 11966. https://doi.org/10.1038/ncomms11966.

(9) Yao, M.; Goult, B. T.; Chen, H.; Cong, P.; Sheetz, M. P.; Yan, J. Mechanical Activation of Vinculin Binding to Talin Locks Talin in an Unfolded Conformation. Scientific Reports 2014, 4 (1), 4610. https://doi.org/10.1038/srep04610. 
(10) Goult, B. T.; Zacharchenko, T.; Bate, N.; Tsang, R.; Hey, F.; Gingras, A. R.; Elliott, P. R.; Roberts, G. C. K.; Ballestrem, C.; Critchley, D. R.; Barsukov, I. L. RIAM and Vinculin Binding to Talin Are Mutually Exclusive and Regulate Adhesion Assembly and Turnover *. Journal of Biological Chemistry 2013, 288 (12), 8238-8249. https://doi.org/10.1074/jbc.M112.438119.

(11) Langer, T.; Lu, C.; Echols, H.; Flanagan, J.; Hayer, M. K.; Hartl, F. U. Successive Action of DnaK, DnaJ and GroEL along the Pathway of Chaperone-Mediated Protein Folding. Nature 1992, 356 (6371), 683-689. https://doi.org/10.1038/356683a0.

(12) Avellaneda, M. J.; Koers, E. J.; Naqvi, M. M.; Tans, S. J. The Chaperone Toolbox at the Single-molecule Level: From Clamping to Confining. Protein Sci 2017, 26 (7), 1291-1302. https://doi.org/10.1002/pro.3161.

(13) Fujita, Y.; Ohto, E.; Katayama, E.; Atomi, Y. AlphaB-Crystallin-Coated MAP Microtubule Resists Nocodazole and Calcium-Induced Disassembly. J Cell Sci 2004, 117 (Pt 9), 1719-1726. https://doi.org/10.1242/jcs.01021.

(14) Pereira, M. B. M.; Santos, A. M.; Gonçalves, D. C.; Cardoso, A. C.; Consonni, S. R.; Gozzo, F. C.; Oliveira, P. S.; Pereira, A. H. M.; Figueiredo, A. R.; Tiroli-Cepeda, A. O.; Ramos, C. H. I.; de Thomaz, A. A.; Cesar, C. L.; Franchini, K. G. AB-Crystallin Interacts with and Prevents Stress-Activated Proteolysis of Focal Adhesion Kinase by Calpain in Cardiomyocytes. Nature Communications 2014, 5 (1), 5159. https://doi.org/10.1038/ncomms6159.

(15) Bullard, B.; Ferguson, C.; Minajeva, A.; Leake, M. C.; Gautel, M.; Labeit, D.; Ding, L.; Labeit, S.; Horwitz, J.; Leonard, K. R.; Linke, W. A. Association of the Chaperone AlphaB-Crystallin with Titin in Heart Muscle. J Biol Chem 2004, 279 (9), 7917-7924. https://doi.org/10.1074/jbc.M307473200.

(16) Shimizu, M.; Tanaka, M.; Atomi, Y. Small Heat Shock Protein AB-Crystallin Controls Shape and Adhesion of Glioma and Myoblast Cells in the Absence of Stress. PLOS ONE 2016, 11 (12), e0168136. https://doi.org/10.1371/journal.pone.0168136.

(17) Collier, M. P.; Benesch, J. L. P. Small Heat-Shock Proteins and Their Role in Mechanical Stress. Cell Stress Chaperones 2020, 25 (4), 601-613. https://doi.org/10.1007/s12192-020-01095-z.

(18) Chakraborty, S.; Chaudhuri, D.; Chaudhuri, D.; Singh, V.; Banerjee, S.; Haldar, S. Real-Time Microfluidics-Magnetic Tweezers Connects Conformational Stiffness with Energy Landscape by a Single Experiment. bioRxiv 2020, 2020.06.09.142257. https://doi.org/10.1101/2020.06.09.142257.

(19) Haining, A. W. M.; von Essen, M.; Attwood, S. J.; Hytönen, V. P.; del Río Hernández, A. All Subdomains of the Talin Rod Are Mechanically Vulnerable and May Contribute To Cellular Mechanosensing. ACS Nano 2016, 10 (7), 6648-6658. https://doi.org/10.1021/acsnano.6b01658.

(20) Tapia-Rojo, R.; Alonso-Caballero, Á.; Fernández, J. M. Talin Folding as the Tuning Fork of Cellular Mechanotransduction. Proceedings of the National Academy of Sciences of the United States of America 2020, 117 (35), 21346-21353. https://doi.org/10.1073/pnas.2004091117.

(21) Tapia-Rojo, R.; Alonso-Caballero, A.; Fernandez, J. M. Direct Observation of a Coilto-Helix Contraction Triggered by Vinculin Binding to Talin. Science Advances 2020, 6 (21), eaaz4707. https://doi.org/10.1126/sciadv.aaz4707.

(22) Popa, I.; Rivas-Pardo, J. A.; Eckels, E. C.; Echelman, D. J.; Badilla, C. L.; ValleOrero, J.; Fernández, J. M. A HaloTag Anchored Ruler for Week-Long Studies of Protein Dynamics. J. Am. Chem. Soc. 2016, 138 (33), 10546-10553. https://doi.org/10.1021/jacs.6b05429. 
(23) Nunes, J. M.; Mayer-Hartl, M.; Hartl, F. U.; Müller, D. J. Action of the Hsp70 Chaperone System Observed with Single Proteins. Nature Communications 2015, 6 (1), 6307. https://doi.org/10.1038/ncomms7307.

(24) Eckels, E. C.; Chaudhuri, D.; Chakraborty, S.; Echelman, D. J.; Haldar, S. DsbA Is a Redox-Switchable Mechanical Chaperone. bioRxiv 2021, 310169. https://doi.org/10.1101/310169.

(25) Yan, J.; Yao, M.; Goult, B. T.; Sheetz, M. P. Talin Dependent Mechanosensitivity of Cell Focal Adhesions. Cell Mol Bioeng 2015, 8 (1), 151-159. https://doi.org/10.1007/s12195-014-0364-5.

(26) Imamoglu, R.; Balchin, D.; Hayer-Hartl, M.; Hartl, F. U. Bacterial Hsp70 Resolves Misfolded States and Accelerates Productive Folding of a Multi-Domain Protein. Nature Communications 2020, 11 (1), 365. https://doi.org/10.1038/s41467-019-142454.

(27) Schröder, H.; Langer, T.; Hartl, F. U.; Bukau, B. DnaK, DnaJ and GrpE Form a Cellular Chaperone Machinery Capable of Repairing Heat-Induced Protein Damage. EMBO J 1993, 12 (11), 4137-4144.

(28) Szabo, A.; Langer, T.; Schröder, H.; Flanagan, J.; Bukau, B.; Hartl, F. U. The ATP Hydrolysis-Dependent Reaction Cycle of the Escherichia Coli Hsp70 System DnaK, DnaJ, and GrpE. Proc Natl Acad Sci U S A 1994, 91 (22), 10345-10349. https://doi.org/10.1073/pnas.91.22.10345.

(29) Mayer, M. P. Hsp70 Chaperone Dynamics and Molecular Mechanism. Trends Biochem Sci 2013, 38 (10), 507-514. https://doi.org/10.1016/j.tibs.2013.08.001.

(30) Clerico, E. M.; Tilitsky, J. M.; Meng, W.; Gierasch, L. M. How Hsp70 Molecular Machines Interact with Their Substrates to Mediate Diverse Physiological Functions. $J$ Mol Biol 2015, 427 (7), 1575-1588. https://doi.org/10.1016/j.jmb.2015.02.004.

(31) Lagarrigue, F.; Vikas Anekal, P.; Lee, H.-S.; Bachir, A. I.; Ablack, J. N.; Horwitz, A. F.; Ginsberg, M. H. A RIAM/Lamellipodin-Talin-Integrin Complex Forms the Tip of Sticky Fingers That Guide Cell Migration. Nature Communications 2015, 6 (1), 8492. https://doi.org/10.1038/ncomms9492.

(32) Rahikainen, R.; von Essen, M.; Schaefer, M.; Qi, L.; Azizi, L.; Kelly, C.; Ihalainen, T. O.; Wehrle-Haller, B.; Bastmeyer, M.; Huang, C.; Hytönen, V. P. Mechanical Stability of Talin Rod Controls Cell Migration and Substrate Sensing. Scientific Reports 2017, 7 (1), 3571. https://doi.org/10.1038/s41598-017-03335-2.

(33) Kumar, A.; Ouyang, M.; Van den Dries, K.; McGhee, E. J.; Tanaka, K.; Anderson, M. D.; Groisman, A.; Goult, B. T.; Anderson, K. I.; Schwartz, M. A. Talin Tension Sensor Reveals Novel Features of Focal Adhesion Force Transmission and Mechanosensitivity. Journal of Cell Biology 2016, 213 (3), 371-383. https://doi.org/10.1083/jcb.201510012.

(34) Grashoff, C.; Hoffman, B. D.; Brenner, M. D.; Zhou, R.; Parsons, M.; Yang, M. T.; McLean, M. A.; Sligar, S. G.; Chen, C. S.; Ha, T.; Schwartz, M. A. Measuring Mechanical Tension across Vinculin Reveals Regulation of Focal Adhesion Dynamics. Nature 2010, 466 (7303), 263-266. https://doi.org/10.1038/nature09198.

(35) Kenific, C. M.; Wittmann, T.; Debnath, J. Autophagy in Adhesion and Migration. Journal of Cell Science 2016, $129 \quad$ (20), 3685-3693. https://doi.org/10.1242/jcs.188490.

(36) Ulbricht, A.; Eppler, F. J.; Tapia, V. E.; van der Ven, P. F. M.; Hampe, N.; Hersch, N.; Vakeel, P.; Stadel, D.; Haas, A.; Saftig, P.; Behrends, C.; Fürst, D. O.; Volkmer, R.; Hoffmann, B.; Kolanus, W.; Höhfeld, J. Cellular Mechanotransduction Relies on Tension-Induced and Chaperone-Assisted Autophagy. Curr Biol 2013, 23 (5), 430435. https://doi.org/10.1016/j.cub.2013.01.064. 
(37) Haldar, S.; Tapia-Rojo, R.; Eckels, E. C.; Valle-Orero, J.; Fernandez, J. M. Trigger Factor Chaperone Acts as a Mechanical Foldase. Nature Communications 2017, 8 (1), 668. https://doi.org/10.1038/s41467-017-00771-6.

(38) Chaudhuri, D.; Banerjee, S.; Chakraborty, S.; Halder, S. The Mechanical Roles of $\begin{array}{lll}\text { Chaperones. } & \text { bioRxiv 2020.10.20.346973. }\end{array}$ https://doi.org/10.1101/2020.10.20.346973.

(39) Banerjee, R.; Jayaraj, G. G.; Peter, J. J.; Kumar, V.; Mapa, K. Monitoring Conformational Heterogeneity of the Lid of DnaK Substrate-Binding Domain during Its Chaperone Cycle. FEBS J 2016, $283 \quad$ (15), 2853-2868. https://doi.org/10.1111/febs.13769.

(40) Kahn, T. B.; Fernández, J. M.; Perez-Jimenez, R. Monitoring Oxidative Folding of a Single Protein Catalyzed by the Disulfide Oxidoreductase DsbA*. Journal of Biological Chemistry 2015, $290 \quad$ (23), $14518-14527$. https://doi.org/10.1074/jbc.M115.646000.

(41) Valle-Orero, J.; Tapia-Rojo, R.; Eckels, E. C.; Rivas-Pardo, J. A.; Popa, I.; Fernández, J. M. Proteins Breaking Bad: A Free Energy Perspective. J. Phys. Chem. Lett. 2017, 8 (15), 3642-3647. https://doi.org/10.1021/acs.jpclett.7b01509.

(42) Valle-Orero, J.; Rivas-Pardo, J. A.; Tapia-Rojo, R.; Popa, I.; Echelman, D. J.; Haldar, S.; Fernández, J. M. Mechanical Deformation Accelerates Protein Ageing. Angewandte Chemie International Edition 2017, $56 \quad$ (33), 9741-9746. https://doi.org/10.1002/anie.201703630. 


\section{Figures}

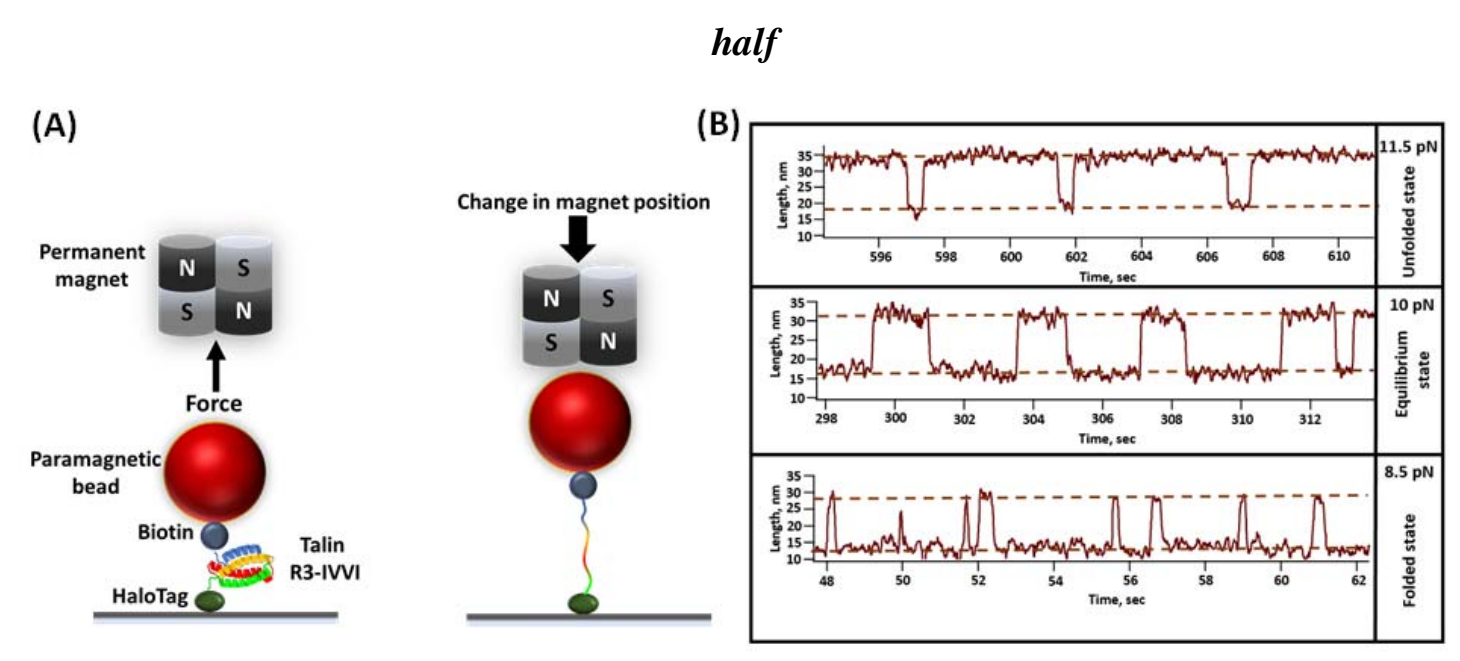

(C)

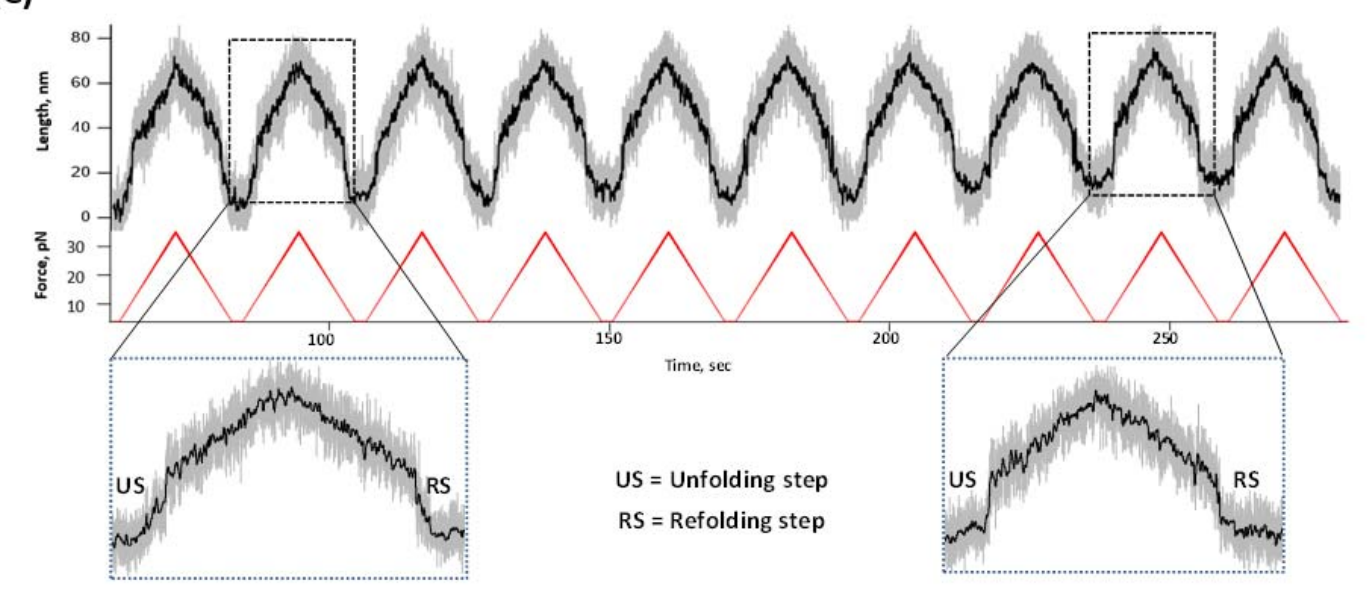

Figure 1: Microfuidic magnetic tweezers set-up to study the folding dynamics and mechanical stability of talin R3-IVVI. (A) Schematic representation of magnetic tweezers experiment: The engineered construct of single R3-IVVI domain is flanked between the glass 
surface and the streptavidin coated paramagnetic bead using halotag chemistry. Applied force is controlled by changing the distance beween paramagnetic bead and permanent magnet. (B)Force-dependent folding dynamics of talin R3-IVVI domain: Talin R3-IVVI exhibits a strong force-dependent folding dynamics which is characterized as reversible folding-unfolding transitions, yielding extension changes of $\sim 20 \mathrm{~nm}$. At $11.5 \mathrm{pN}$ force, it is mostly present in unfolded state with a FP of 0.2 , while at $8.5 \mathrm{pN}$, it is mostly in the folded state (FP 0.92). At $10 \mathrm{pN}, \mathrm{FP}$ is 0.52, where both the states occupy almost equal population. (C) Force ramp study of R3-IVVI: Successive force ramp, from $4 \mathrm{pN}$ to $35 \mathrm{pN}$ at a constant rate of 3.1pN/sec, are applied to observe distinct unfolding and refolding steps at particular forces. The traces are magnified to observe the mechanical stability of the domain throughout the successive force ramp experiment.

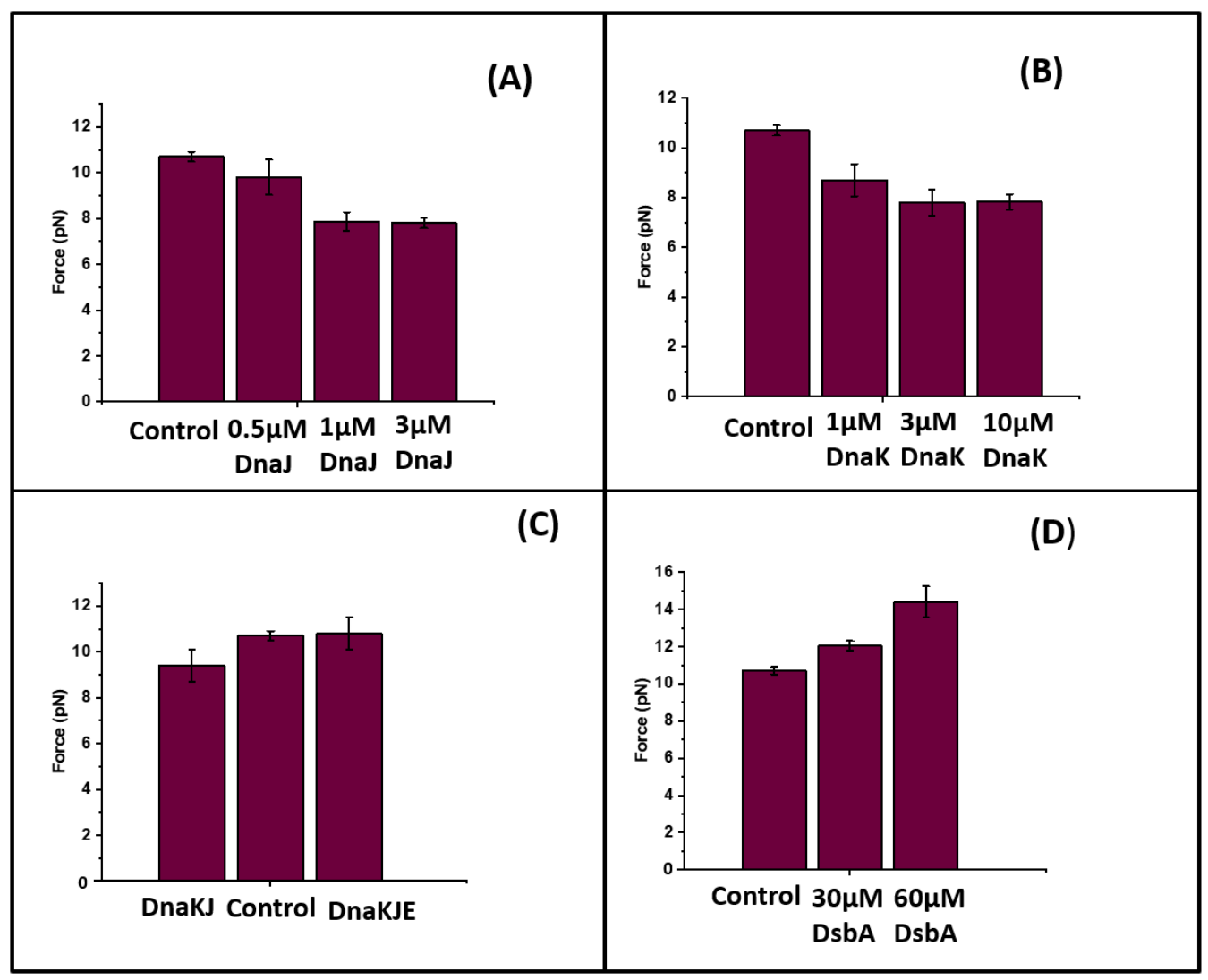

Figure 2: Force ramp experiment detects the chaperone-mediated change in unfolding force of talin R3-IVVI domain: In an unfolding force ramp protocol, the force is increased from $4 \mathrm{pN}$ to $35 \mathrm{pN}$ at a constant rate of $3.1 \mathrm{pN} / \mathrm{sec}$. (A) Effect of DnaJ: Unfolding event in the absence of chaperones (control) is observed at 10.7 $\pm 0.21 \mathrm{pN}$, which has been observed to decrease steadily with DnaJ concentration. Data points are measured by using more than six individual protein molecules. Error bars are standard error of mean (s.e.m.). (B)Effect of DnaK: In the presence of DnaK, R3-IVVI unfolds at lower force and the force decreases with the increasing concentration of DnaK. Data points are calculated using more than eight protein molecules. Error bars are s.e.m. (C) Effect of DnaKJ and DnaKJE: In the presence of DnaK, DnaJ and ATP, R3-IVVI unfolds at lower force of 9.4 $\pm 0.7 \mathrm{pN}$, while upon adding GrpE (DnaK+ DnaJ+ GrpE+ ATP), it unfolds at a similar force with the control. These 
experiments have been done in $10 \mathrm{mM}$ ATP and $10 \mathrm{mM} \mathrm{MgCl}_{2}$. The buffer is changed after every 30 minutes with fresh ATP to keep the sufficient supply of ATP. Data points are measured using more than seven molecules. Error bars are s.e.m. (D) Effect of DsbA: DsbA increases the unfolding force in force ramp experiments. R3-IVVI domain unfolds at $10.7 \pm 0.21 \mathrm{pN}$ force whereas it increases to $12.05 \pm 0.25 \mathrm{pN}$ with $30 \mu \mathrm{M} \mathrm{Ds} b \mathrm{~A}$ and reaches to $14.74 \pm 0.85 \mathrm{pN}$ in the presence of $60 \mu \mathrm{M}$ DsbA. Data points are measured using more than five molecules. Error bar are represented as s.e.m.

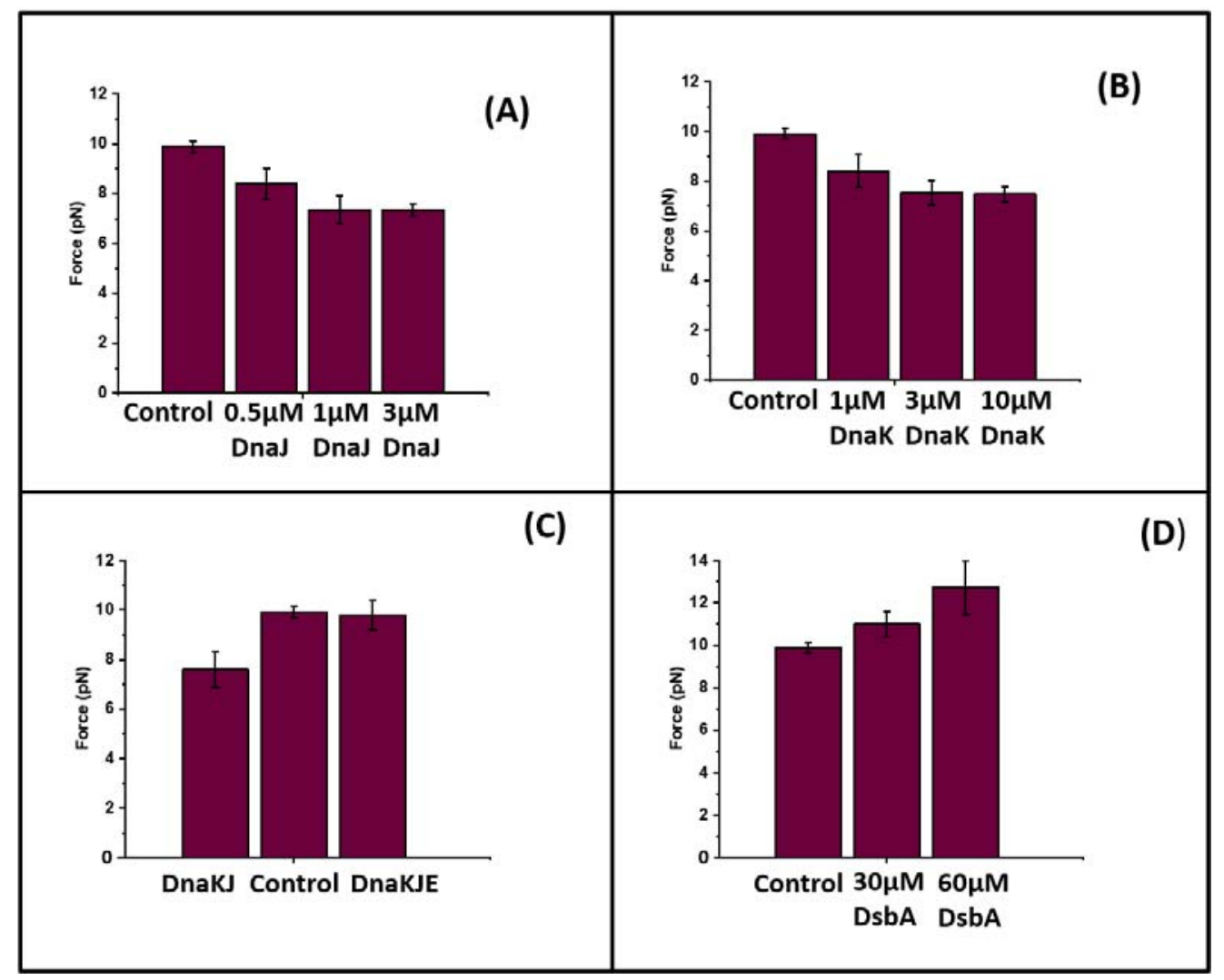

Figure 3: Force ramp protocol detects the chaperon-mediated change in refolding force of R3-IVVI: In a refolding force ramp protocol, the force is decreased from $35 \mathrm{pN}$ to $4 \mathrm{pN}$ at a rate of 3.1pN/sec. (A) Refolding force in the presence of DnaJ: In the absence of DnaJ, the refolding event of R3-IVVI is observed at $9.9 \pm 0.23 \mathrm{pN}$ and with the gradual addition of DnaJ,

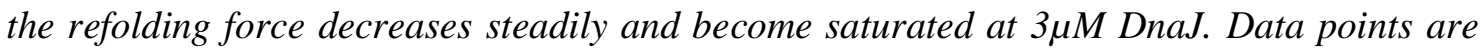
calculated using more than eight individual protein molecules. Error bars are s.e.m. (B) Effect of DnaK: Similar to DnaJ, DnaK decreases the refolding force of R3-IVVI. Data points are calculated by using more than seven individual protein molecules. Error bars are s.e.m. (C) Effect of DnaKJ and DnaKJE on the refolding force of R3-IVVI: In the presence of DnaKJ and ATP, R3-IVVI refolds at lower force (7.56 $\pm 0.7 \mathrm{pN})$ than control $(9.9 \pm 0.23$ $p N)$, whereas upon addition of GrpE to this mixture (DnaK, DnaJ, GrpE and ATP), it refolds at $9.78 \pm 0.6 \mathrm{pN}$. The buffer is changed after every 30 minutes with fresh ATP to keep the sufficient supply of ATP. Data points are calculated by using more than six protein molecules. Error bars are s.e.m. (D) Refolding of R3-IVVI in the presence of DsbA: The refolding force has been observed to increase from $9.9 \pm 0.23 \mathrm{pN}$ (control) to $11 \pm 0.6 \mathrm{pN}$ in the 
presence of $30 \mu \mathrm{M}$ DsbA, and increases further to $12.74 \pm 1.2 \mathrm{pN}$ with $60 \mu \mathrm{M}$ DsbA. Data points are calculated using more than seven protein molecules. Error bars are represented as s.e.m.

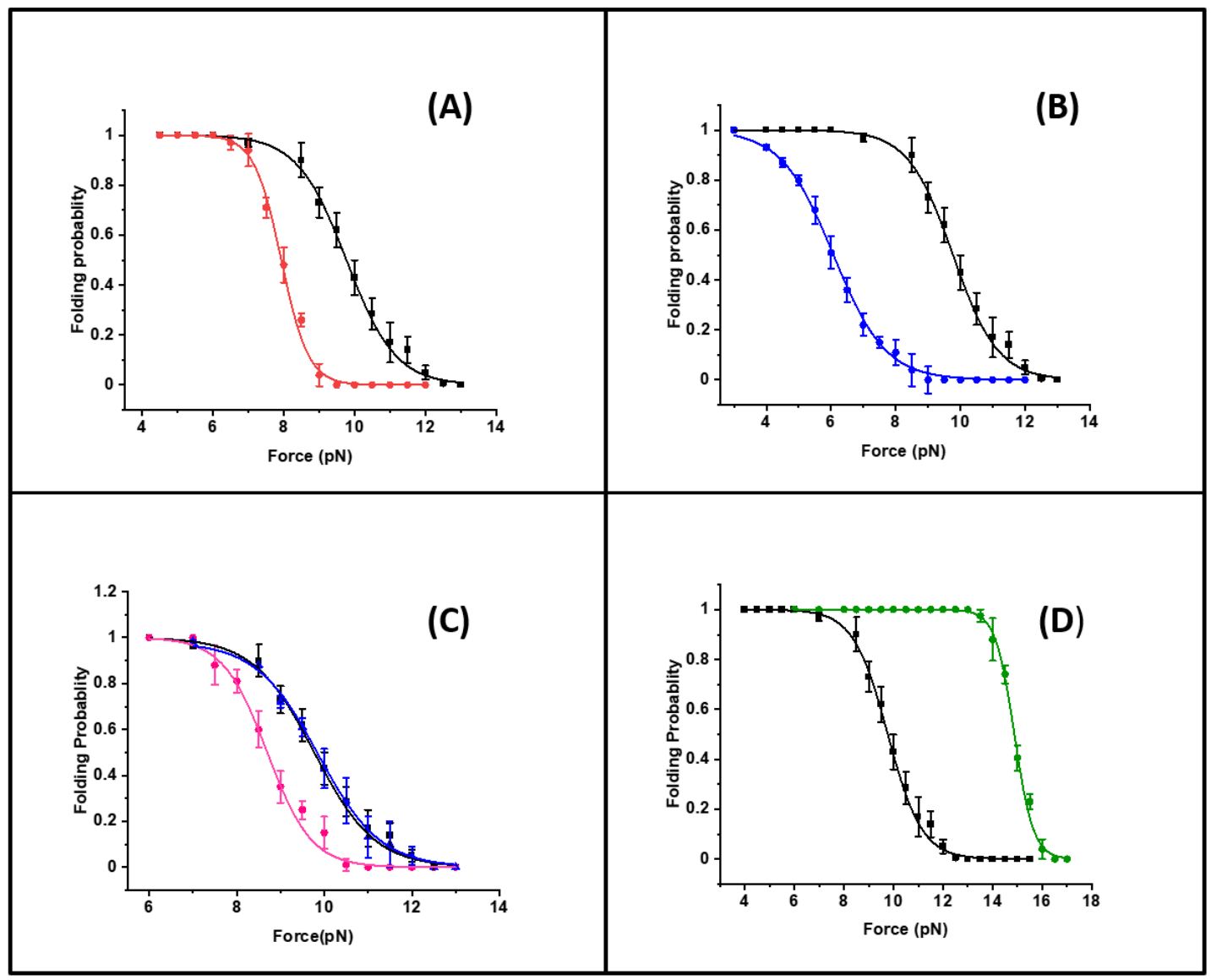

Figure 4: Chaperones modulate the folding probability of talin R3-IVVI: (A) Effect of DnaJ on the folding probability of R3-IVVI: DnaJ shifts the folding probability towards lower force regime and the half-point force has also been observed to shift from 9.76 to 7.9 $p N$, in the presence of $1 \mu M$ DnaJ. Data points are calculated using more than six individual protein molecules. Error bars are standard error by mean. (B)Folding probability in the presence of DnaK: DnaK also reduces the folding probability and in the presence of $3 \mu M$ DnaK, the half-point force shifts from 9.76 to $6.07 \mathrm{pN}$. Data points are calculated by using more than eight individual protein molecules. Error bars are standard by mean. (B) Effect of DnaKJ and DnaKJE complex on the folding probability: In the presence of DnaKJ complex and ATP, the half-point force decreases to $8.69 \mathrm{pN}$ (pink), while upon adding GrpE and ATP, it reverts to $9.86 \mathrm{pN}$ (blue). These experiments has been done in $10 \mathrm{mM}$ ATP and $10 \mathrm{mM}$ $\mathrm{MgCl2}$. The buffer is changed after every 30 minutes with fresh ATP to keep the sufficient supply of ATP. Data points are calculated by using more than six individual protein molecules. Error bars are standard error by mean. (D) Folding probability in the presence of DsbA: DsbA significantly increases the folding probability by shifting the half-point force from 9.76 to 14.87 pN. Data points are calculated using more than six molecules per force. Errors bars are represented as s.e.m. 


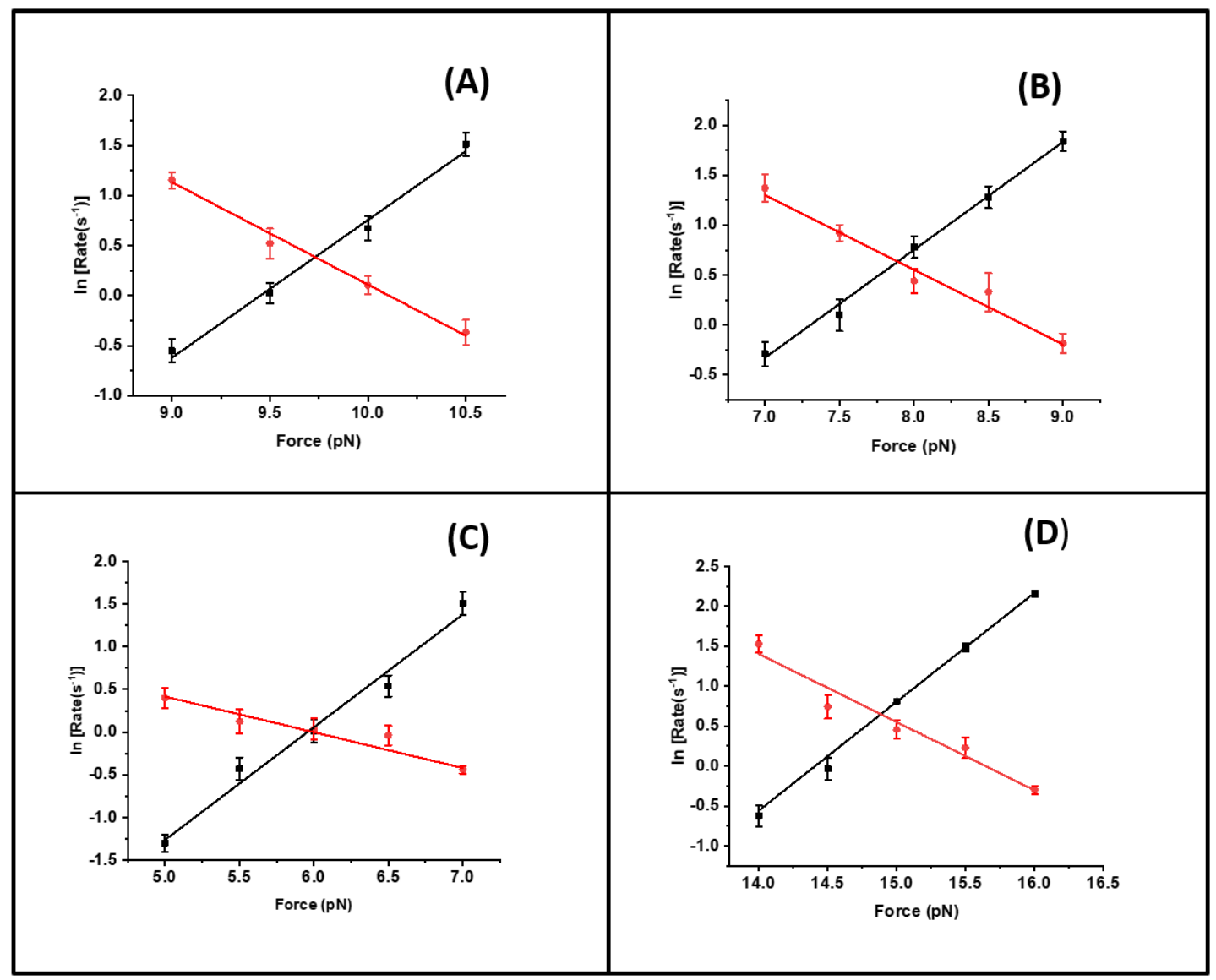

Figure 5: Unfolding and refolding rates of talin R3-IVVI domain: (A) Unfolding and refolding rates in the absence of chaperones (control): Unfolding (black) and refolding (red) rates are plotted as a function of force. (B) Effect of DnaJ on unfolding and refolding rates: Unfolding (black) and refolding (red) rates of talin in the presence of $1 \mu M$ DnaJ. Data points are calculated using more than six protein molecules. Error bars are relative error of log. (C) Effect of DnaK: Refolding (red) and unfolding (black) rates of R3-IVVI at different forces, in the presence of $3 \mu M$ DnaK. Data points are calculated using more than five protein molecules. Error bars are relative error of $\log (\boldsymbol{D})$ Effect of DsbA: Unfolding (black) and refolding (red) rates of R3-IVVI are plotted against the force, in the presence of $60 \mu M D s b A$. Data points are calculated using more than seven protein molecules. Error bars are relative error of $\log$. 


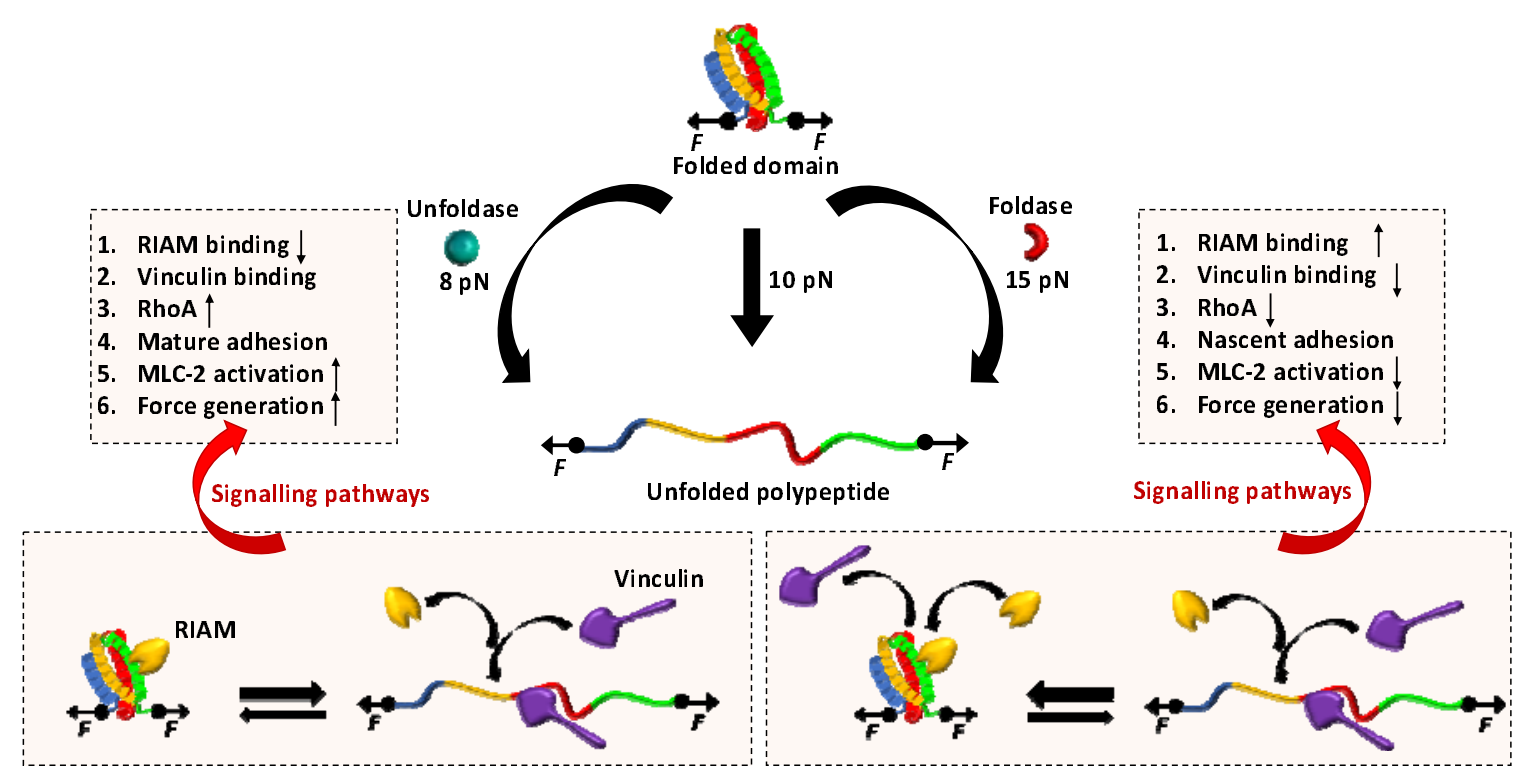

Figure 6: Chaperone-talin interactions modulate downstream signaling pathways: Talin rod domains exhibit strong tension-dependent conformational changes which can further be modulated by both classes of chaperones: unfoldase and foldase. In the absence of chaperones, $R 3$ unfolds at $10 \mathrm{pN}$ force while it shifts to $15 \mathrm{pN}$ or $8 \mathrm{pN}$, in the presence of foldase and unfoldase chaperones, respectively. This altered mechanical stability could influence cellular physiology by tuning the downstream signaling pathways. Unfoldase shifts the folding dynamics of $R 3$ domain towards the unfolded state, thereby, favouring the RIAM dissociation and vinculin interaction. Subsequently, this induces RhoA signaling, downstream $M L C-2$ activation and thus, enhances the force generation at growing adhesion to facilitate cell migration. By contrast, foldase shifts the folding dynamics towards folded state which subsequently promotes RIAM binding to the talin domains and localization in nascent adhesion. This negatively regulates the RhoA pathway which in turn abrogates $M L C-2$ activation, force generation, and the cell migration. 


\begin{tabular}{|c|c|c|c|c|}
\hline \multicolumn{5}{|c|}{ Table 1 } \\
\hline & Control & DnaJ & DnaK & DsbA \\
\hline$\Delta \boldsymbol{G}_{\text {Unfolding }}^{\dagger}$ & $26.8 \pm 0.83 \mathrm{k}_{\mathrm{B}} \mathrm{T}$ & $21.69 \pm 0.28 \mathrm{k}_{\mathrm{B}} \mathrm{T}$ & $21.71 \pm 0.56 \mathrm{k}_{\mathrm{B}} \mathrm{T}$ & $33.46 \pm 0.39 \mathrm{k}_{\mathrm{B}} \mathrm{T}$ \\
\hline$\Delta \boldsymbol{x}_{\text {Unfolding }}$ & $1.37 \pm 0.086 \mathrm{k}_{\mathrm{B}} \mathrm{T}$ & $1.08 \pm 0.034 \mathrm{k}_{\mathrm{B}} \mathrm{T}$ & $1.32 \pm 0.09 \mathrm{k}_{\mathrm{B}} \mathrm{T}$ & $1.36 \pm 0.02 \mathrm{k}_{\mathrm{B}} \mathrm{T}$ \\
\hline
\end{tabular}

Table 1: The parameters are obtained by fitting the unfolding rates to Bell model (see figure 5). $\Delta G^{\dagger}$ Unfolding represents the height of the free energy barrier and $\Delta x_{\text {Unfolding }}$ is the distance from the folded state to the transition state along the reaction coordinate.

\begin{tabular}{|c|c|c|c|c|}
\hline \multicolumn{5}{|c|}{ Table 2 } \\
\hline & Control & DnaJ & DnaK & DsbA \\
\hline$\Delta \boldsymbol{G}_{\text {Refolding }}^{\dagger}$ & $3.49 \pm 0.47 \mathrm{k}_{\mathrm{B}} \mathrm{T}$ & $7.27 \pm 0.43 \mathrm{k}_{\mathrm{B}} \mathrm{T}$ & $11.31 \pm 0.34 \mathrm{k}_{\mathrm{B}} \mathrm{T}$ & $0.44 \pm 0.85 \mathrm{k}_{\mathrm{B}} \mathrm{T}$ \\
\hline$\Delta \boldsymbol{x}_{\text {Refolding }}$ & $0.92 \pm 0.04 \mathrm{k}_{\mathrm{B}} \mathrm{T}$ & $0.75 \pm 0.05 \mathrm{k}_{\mathrm{B}} \mathrm{T}$ & $0.42 \pm 0.05 \mathrm{k}_{\mathrm{B}} \mathrm{T}$ & $0.85 \pm 0.06 \mathrm{k}_{\mathrm{B}} \mathrm{T}$ \\
\hline
\end{tabular}

Table 2: The parameters are obtained by fitting the refolding rates to Bell model. $\Delta G^{\dagger}$ Refolding represents the height of the free energy barrier and $\Delta x_{\text {Refolding }}$ is the distance from unfolded state to the transition state along the reaction coordinate. 


\section{Supplementary Information}

\section{Real time observation of chaperone-modulated talin mechanics under single molecule resolution}

Souradeep Banerjee ${ }^{\dagger}$, Deep Chaudhuri ${ }^{\dagger}$, Soham Chakraborty ${ }^{\dagger}$, Shubhasis Haldar ${ }^{*}$

Department of Biological Sciences, Ashoka University, Sonepat, Haryana 131029

$\dagger$ contributed equally to this work

* to whom correspondence may be addressed. Email- shubhasis.halder@ashoka.edu.in 


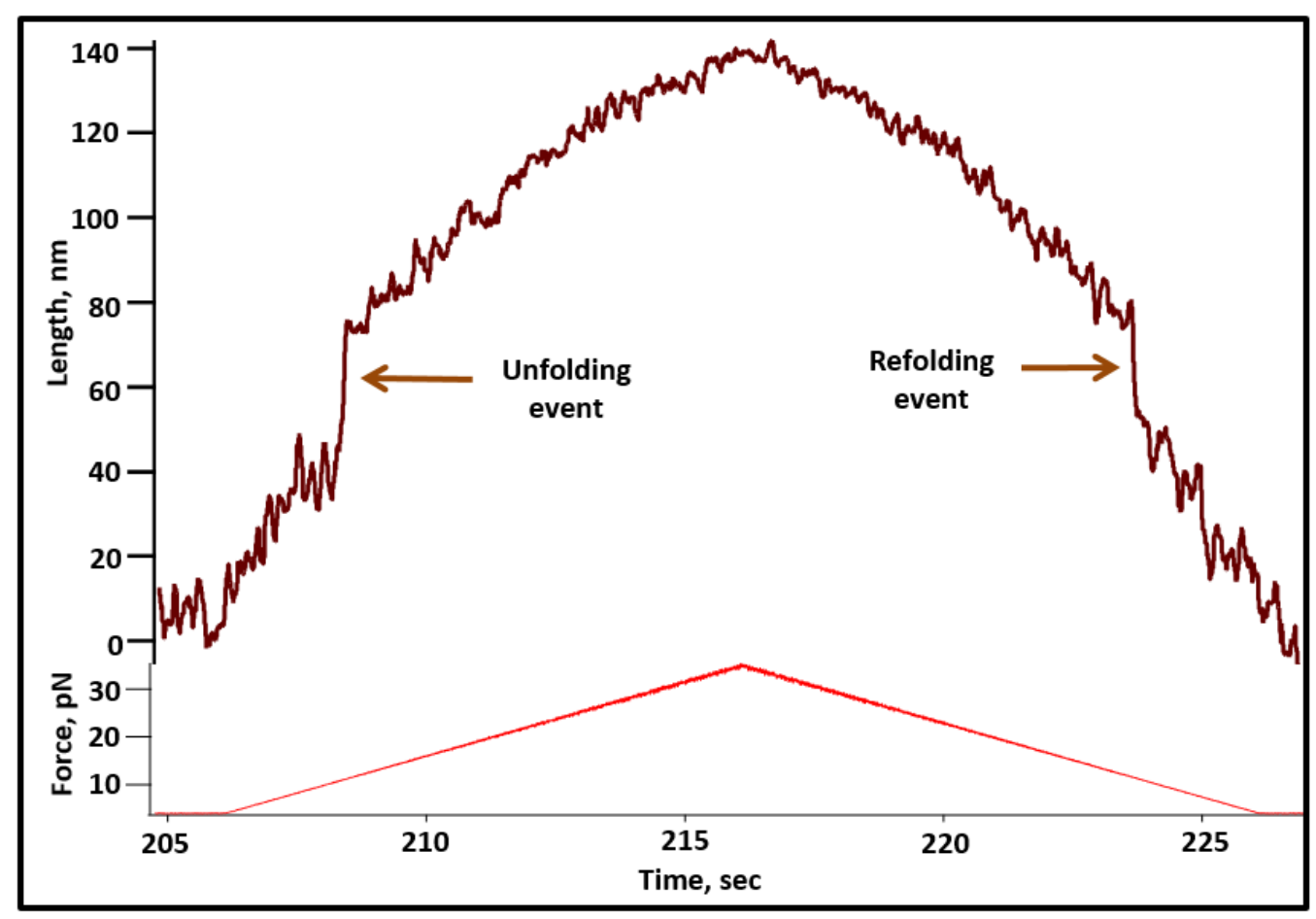

Supplementary figure 1: Representative force ramp trace of talin R3-IVVI: In a force-ramp protocol, at first, the force is increased from 4 to $35 \mathrm{pN}$, at a loading rate of $3.1 \mathrm{pN} / \mathrm{sec}$ and then at the same rate, the force is decreased from 35 to $4 \mathrm{pN}$. The unfolding step was observed at $10.5 \mathrm{pN}$, whereas the refolded step was observed at $10 \mathrm{pN}$. 


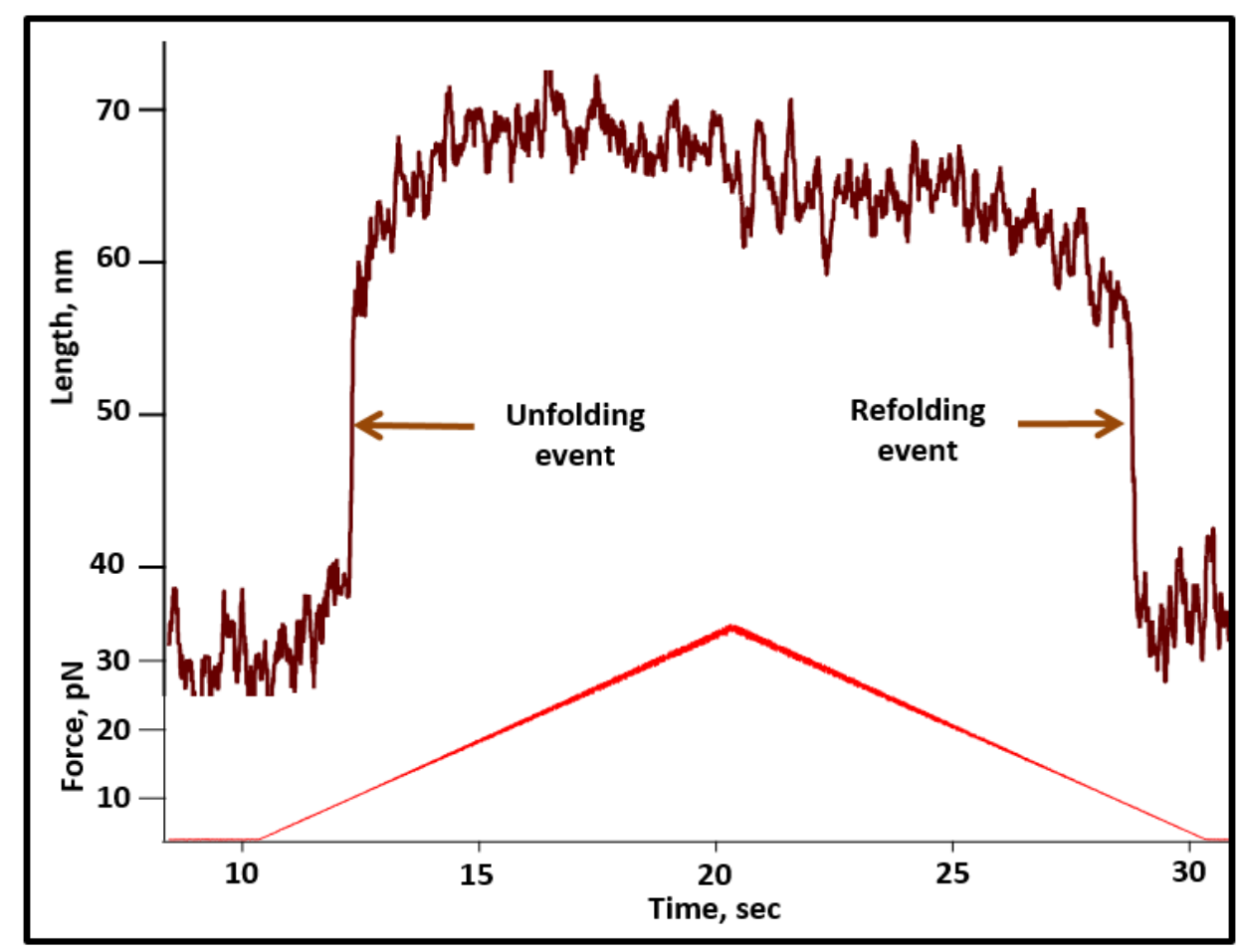

Supplementary figure 2: Representative force-ramp trace of R3-IVVI in presence of 0.5 $\boldsymbol{\mu M}$ DnaJ: During the same force ramp protocol, in the presence of $0.5 \mu M$ DnaJ, unfolding event of R3-IVVI was observed at $9.8 \mathrm{pN}$ and refolding event at $8 \mathrm{pN}$. 


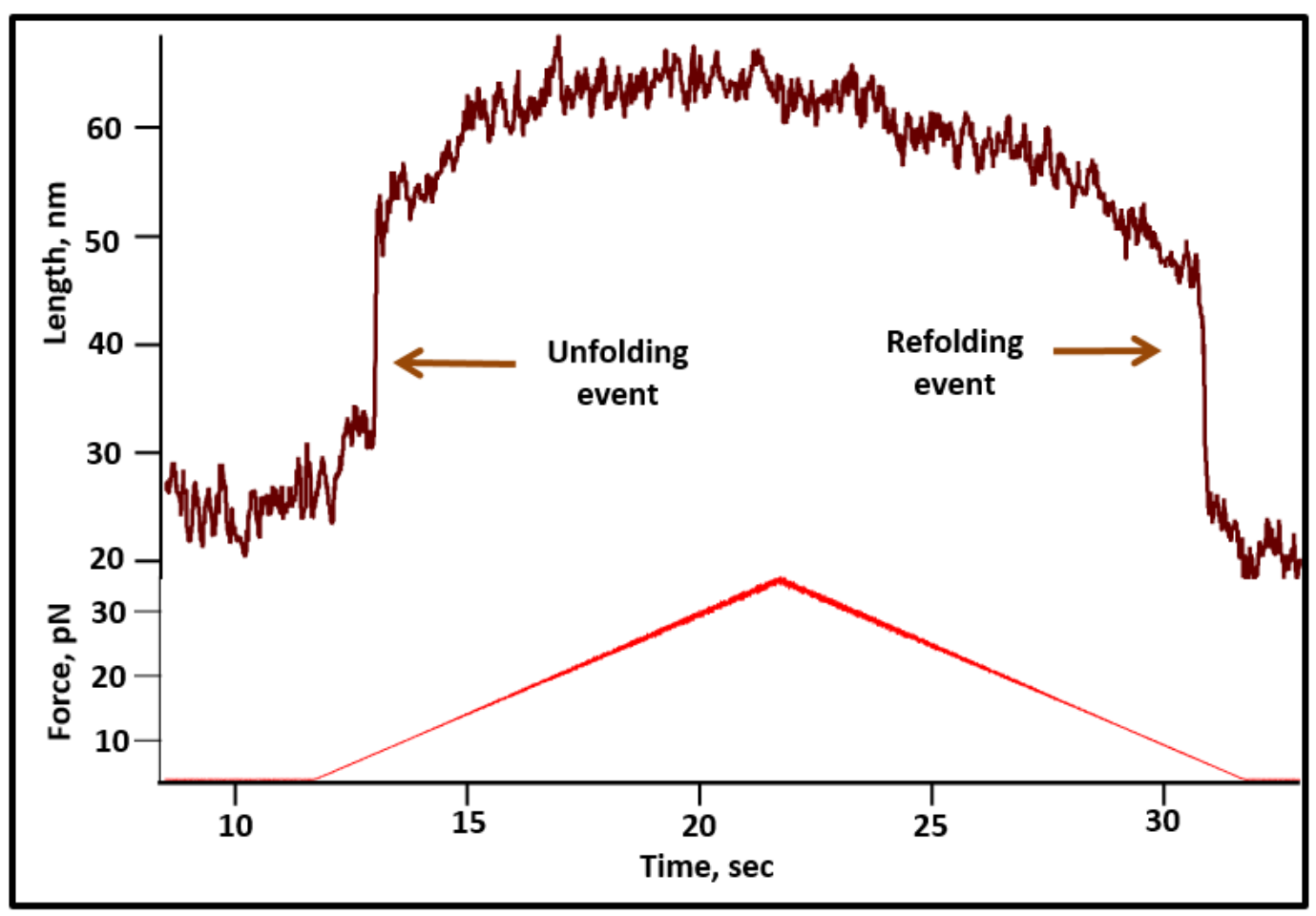

Supplementary figure 3: Representative force ramp trace of R3-IVVI in the presence of $1 \boldsymbol{\mu M}$ DnaJ: During force ramp protocol from 4 to $35 \mathrm{pN}$, at a loading rate of $3.1 \mathrm{pN} / \mathrm{sec}$, R3IVVI unfolds at $8 \mathrm{pN}$. Then the force is decreased gradually to $4 \mathrm{pN}$, where refolding event was observed at $6.5 \mathrm{pN}$. 


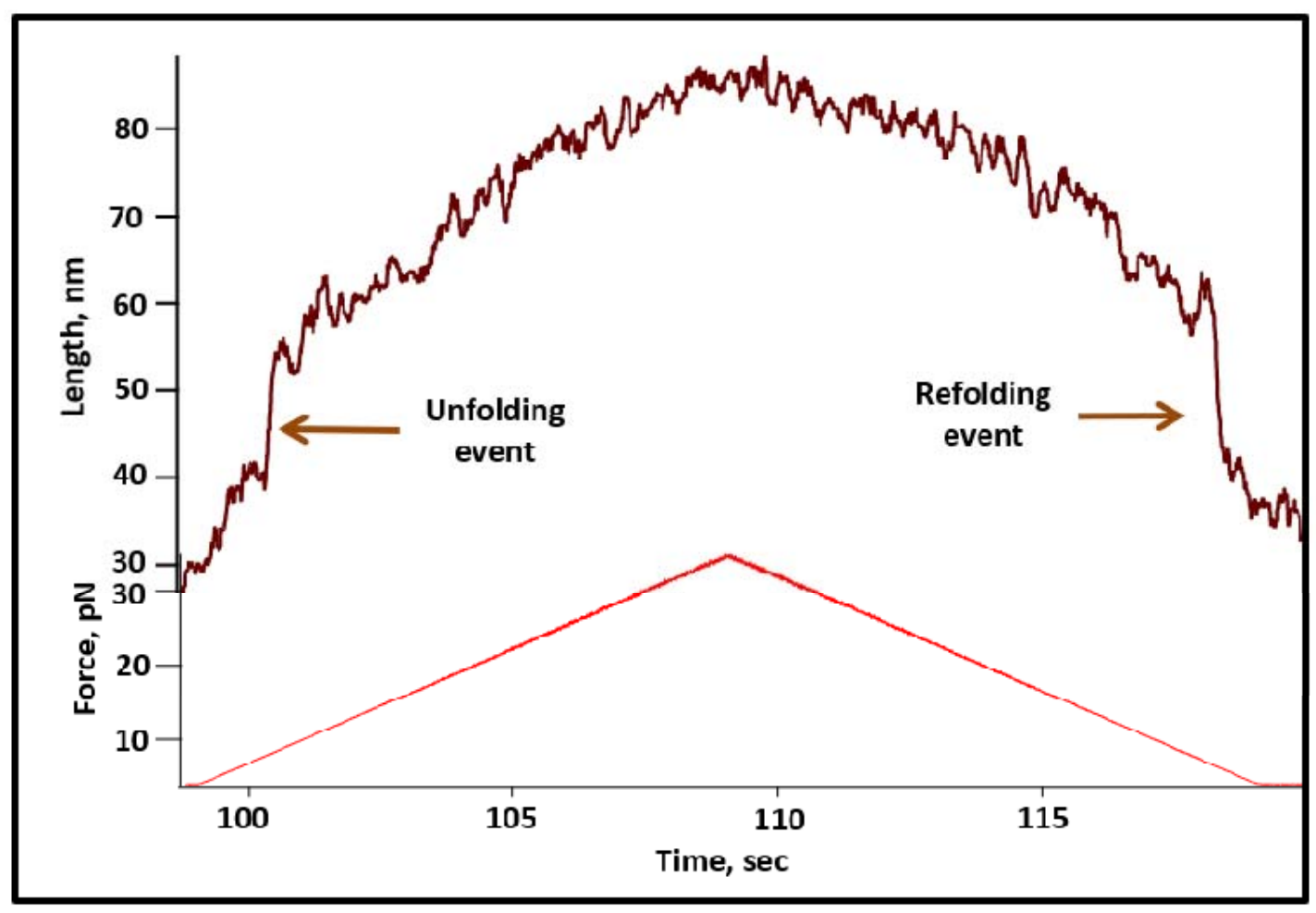

Supplementary figure 4: Representative force ramp trace of talin R3-IVVI in the presence of $3 \boldsymbol{\mu M}$ DnaJ: During force ramp protocol in the presence of $3 \mu M$ DnaJ, talin unfolds at 8.1 $p N$ and refolds at $7 p N$. 


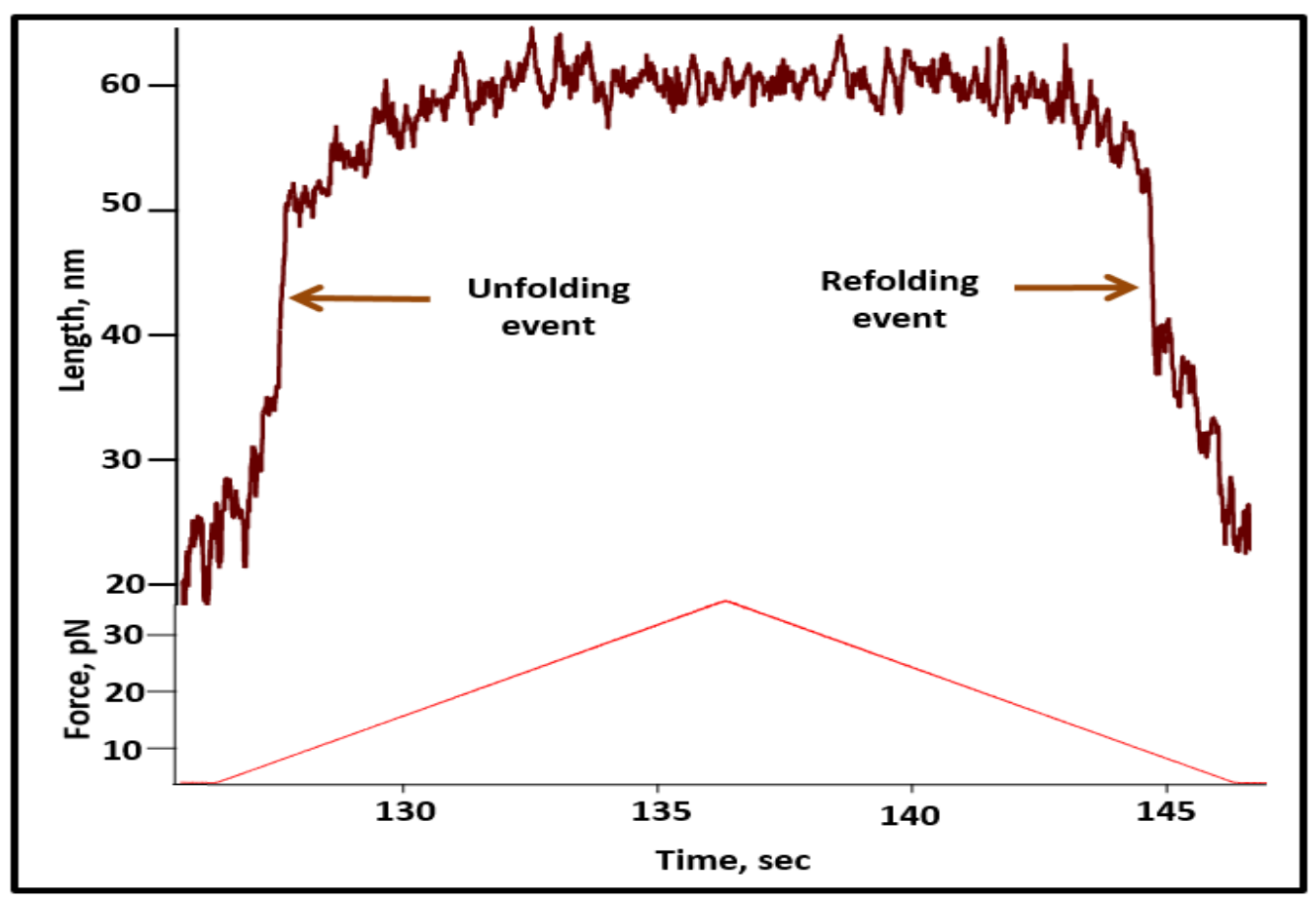

Supplementary figure 5: Representative force-ramp trace of R3-IVVI in the presence of 1.5 $\boldsymbol{\mu M}$ DnaK: Unfolding step at $9 \mathrm{pN}$ was observed by increasing the force from 4 to $35 \mathrm{pN}$ at $3.1 \mathrm{pN} / \mathrm{sec}$. Similarly, refolding step was observed at $10 \mathrm{pN}$ by decreasing the force from 35 to $4 p N$. 


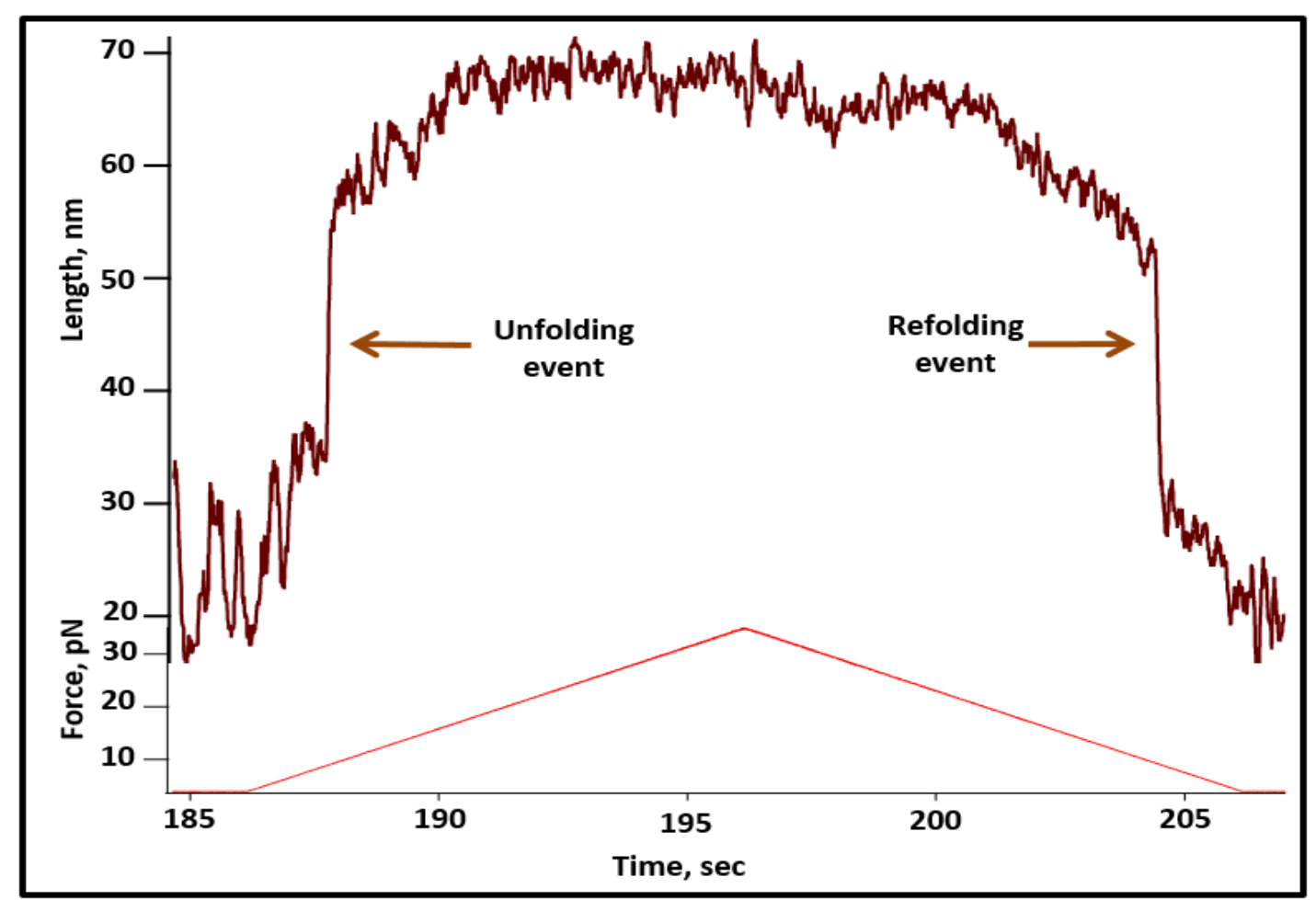

Supplementary figure 6: Representative force-ramp trace of talin R3-IVVI in the presence of $3 \boldsymbol{\mu M}$ DnaK: Here we observed the unfolding and refolding event of talin in the presence of $3 \mu M$ DnaK. Unfolding event observed at $8 p N$ and refolding event at 7.5pN. The force was increased or decreased at a speed of $3.1 \mathrm{pN} / \mathrm{sec}$. 


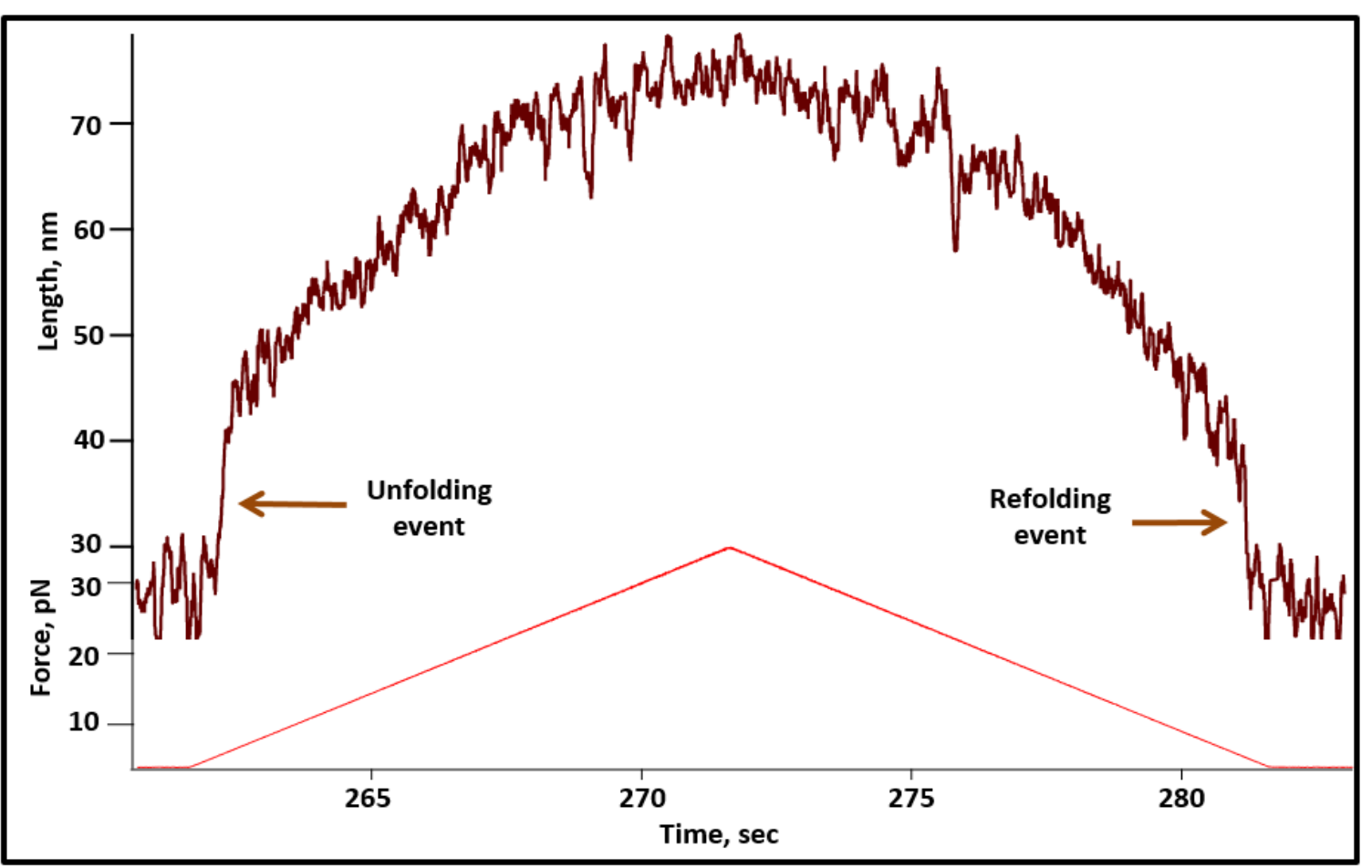

Supplementary figure 7: Representative force-ramp trace of R3-IVVI in the presence of 10 MM DnaK: At first, force was increased from 4 to $35 \mathrm{pN}$ gradually at a speed of $3.1 \mathrm{pN} / \mathrm{sec}$, where the unfolding event was observed at $6.2 \mathrm{pN}$. Then the force decreased from 35 to $4 \mathrm{pN}$ with same loading rate, where refolding event was observed at $6 \mathrm{pN}$. 


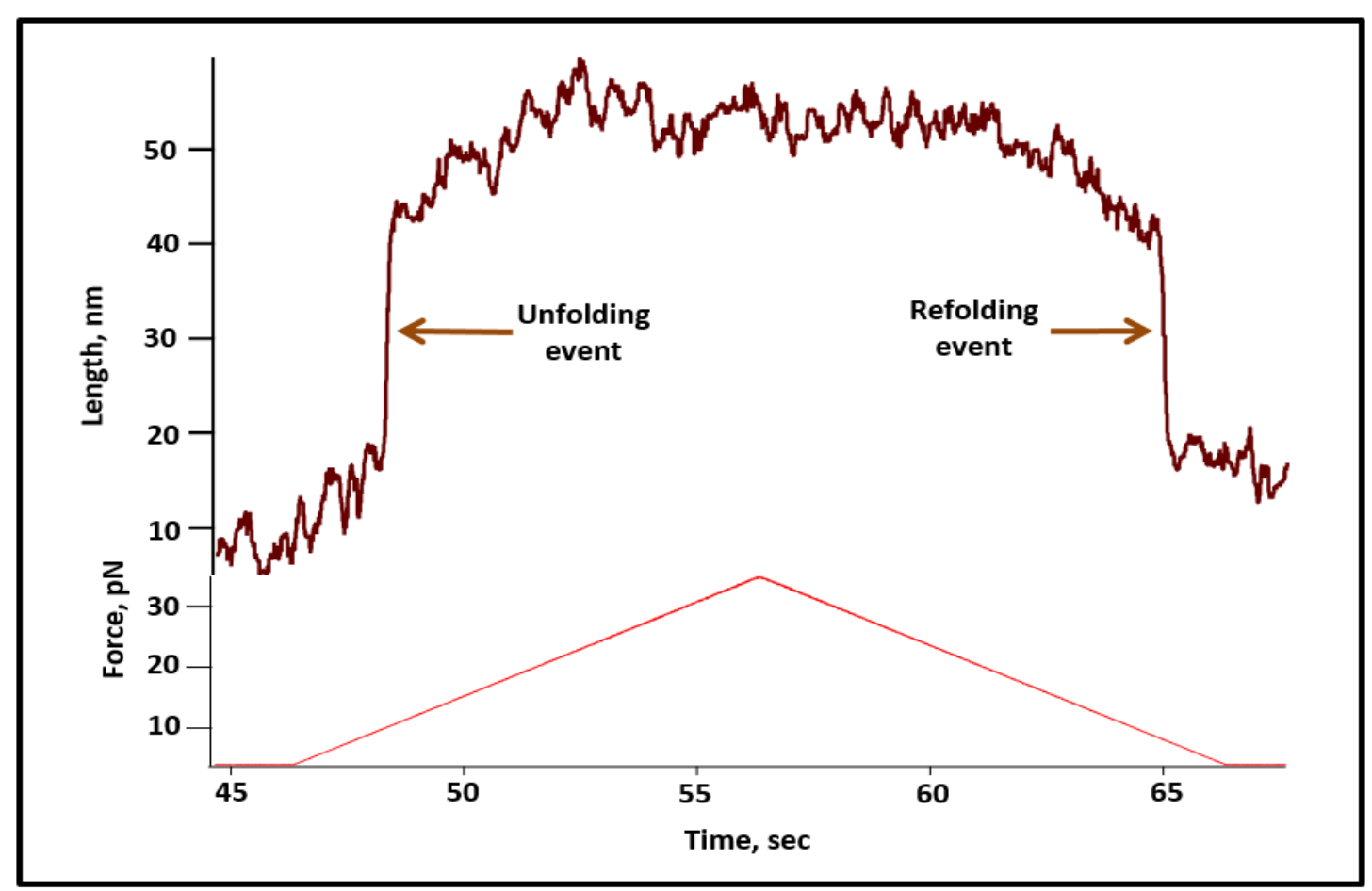

Supplementary figure 8: Representative force-ramp trace of talin talin R3-IVVI in the presence of DnaK, DnaJ and ATP: Unfolding and folding force extension curve of talin with force has been monitored in presence of $1 \mu \mathrm{M}$ DnaJ, $3 \mu \mathrm{M}$ DnaK, $10 \mathrm{mM}$ ATP and $10 \mathrm{mM}$ $\mathrm{MgCl2.The}$ buffer is changed after every 30 minutes with fresh ATP to keep the sufficient supply of ATP. In this condition, unfolding event for talin was observed at $9.7 \mathrm{pN}$ and refolding event was observed at $8.2 \mathrm{pN}$, where the force increased or decreased at a loading rate of $3.1 \mathrm{pN} / \mathrm{sec}$. 


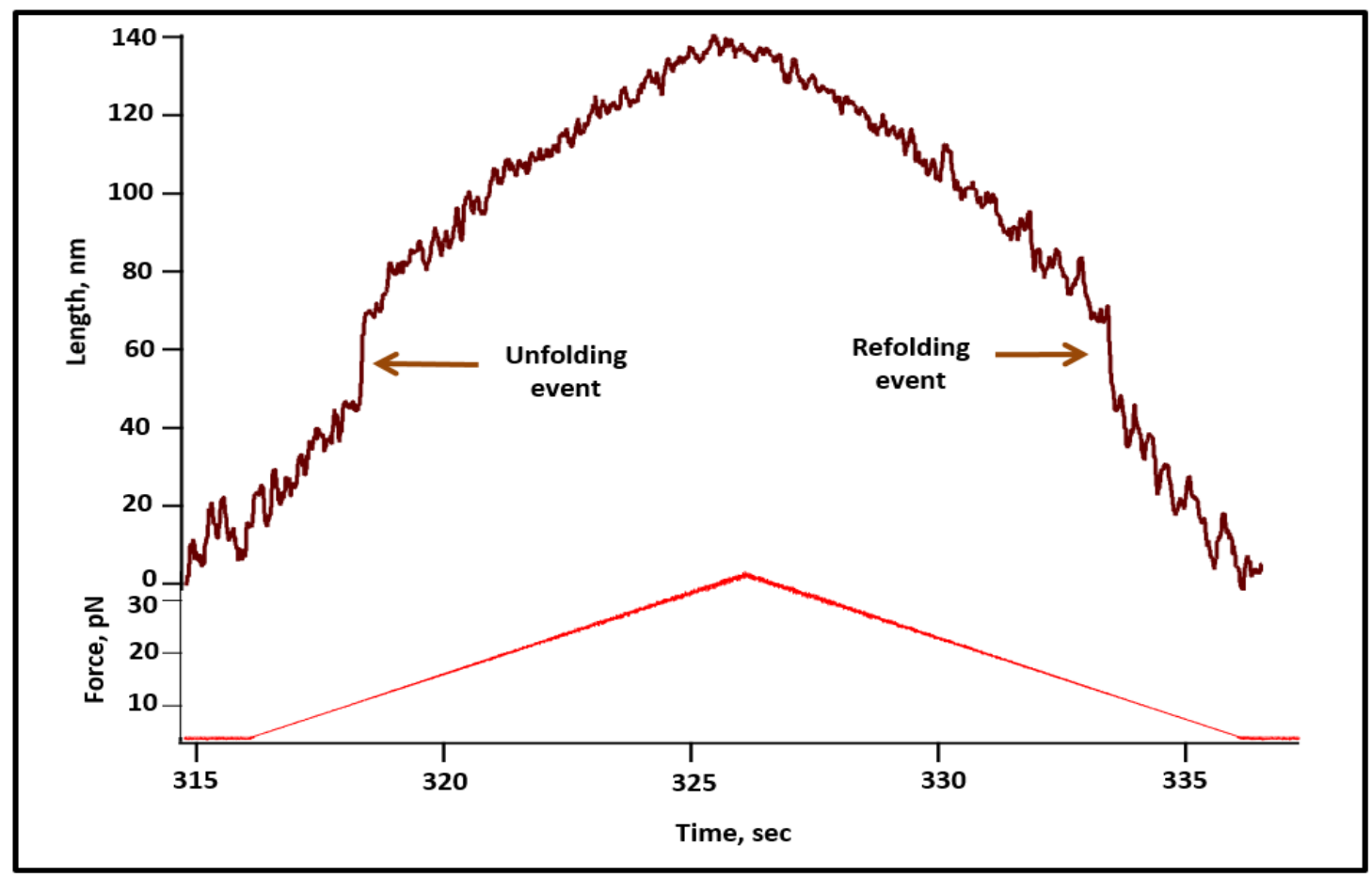

Supplementary figure 9: Representative force-ramp trace of R3-IVVI in the presence of DnaJKE complex: Unfolding and folding events of talin has been monitored in presence of $1 \mu M$ DnaJ, $3 \mu M$ DnaK, $5 \mu M$ GrpE, $10 \mathrm{mM}$ ATP and $10 \mathrm{mM} \mathrm{MgCl}$. The buffer is changed after every 30 minutes with fresh ATP to keep the sufficient supply of ATP. In this condition at a loading rate of $3.1 \mathrm{pN} / \mathrm{sec}$ talin unfolds at 10.8pN and it refolds back at $9.7 \mathrm{pN}$. 


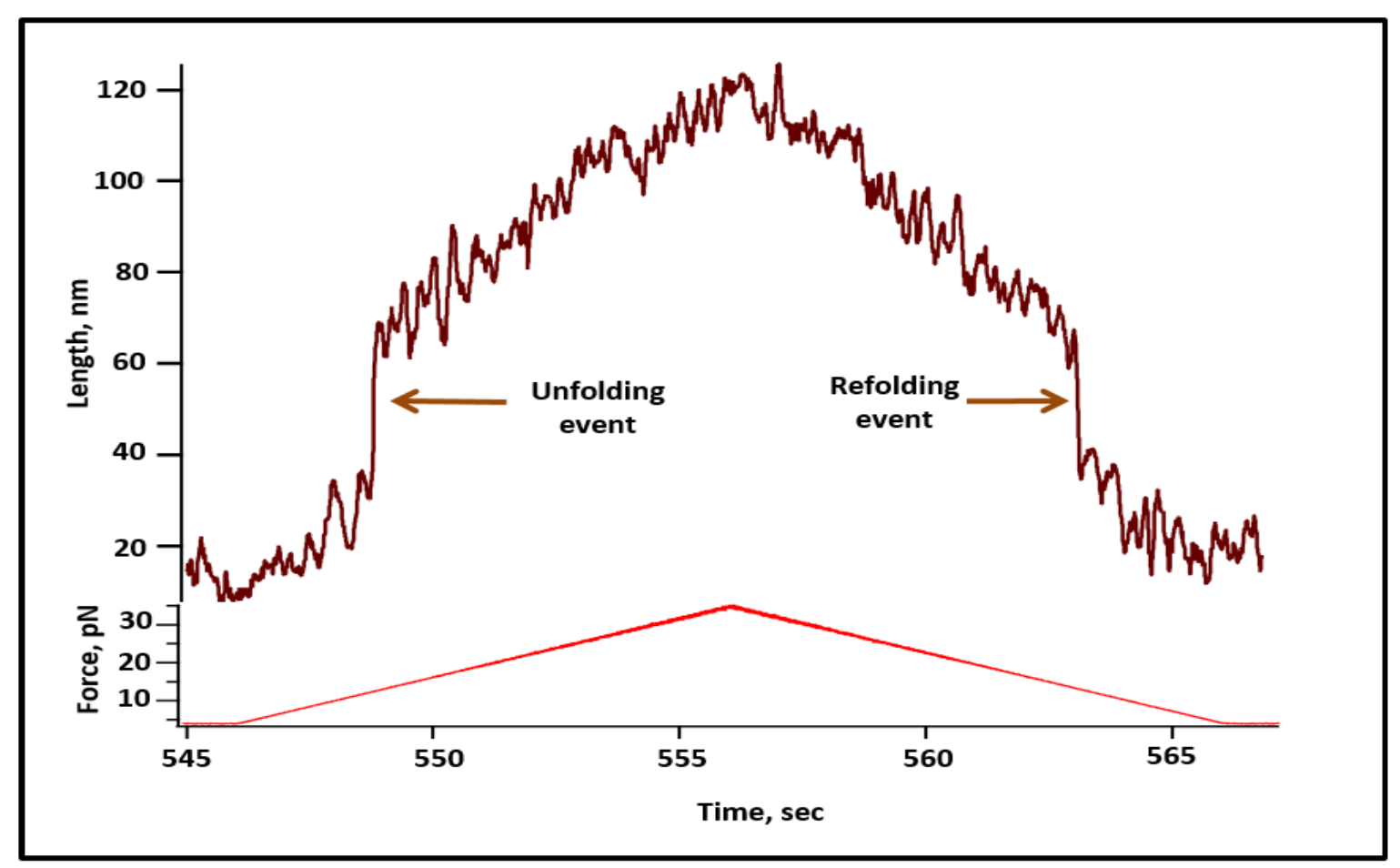

Supplementary figure 10: Representative force ramp trace of talin R3-IVVI in presence of

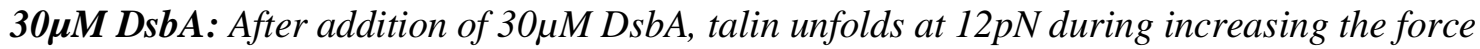
from 4 to $35 \mathrm{pN}$ at a loading rate of $3.1 \mathrm{pN} / \mathrm{sec}$. It refolds at $11 \mathrm{pN}$ when the force decreases from 35 to $4 \mathrm{pN}$ at a same speed of $3.1 \mathrm{pN} / \mathrm{sec}$. 


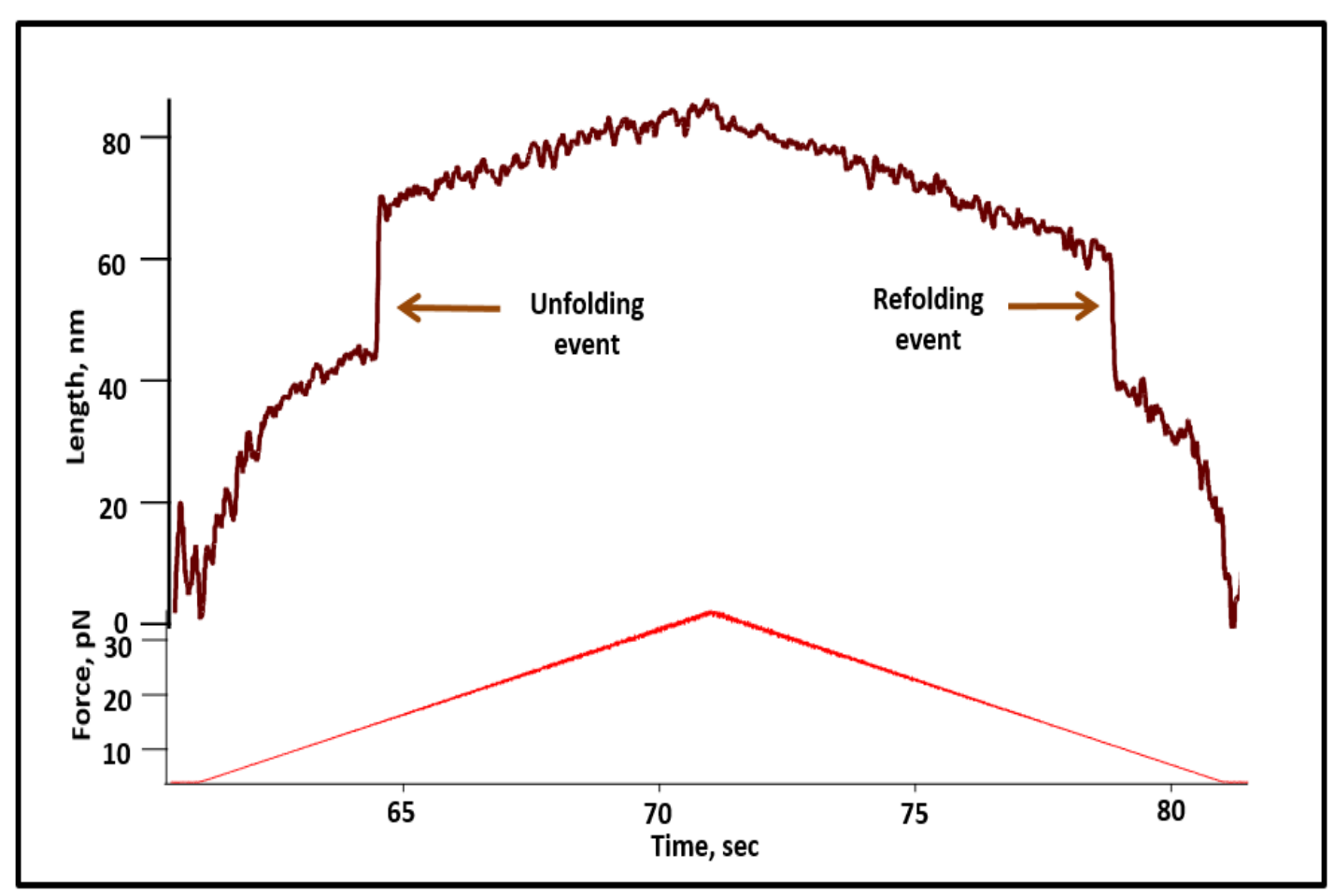

Supplementary figure 11: Representative force-ramp trace of talin in presence of $60 \mu M$ DsbA: In force-ramp protocol the unfolding and refolding event of talin was observed at a loading rate of $3.1 \mathrm{pN} / \mathrm{sec}$. The unfolding event was observed at $14 \mathrm{pN}$ and it refolds back at $11.5 p N$.

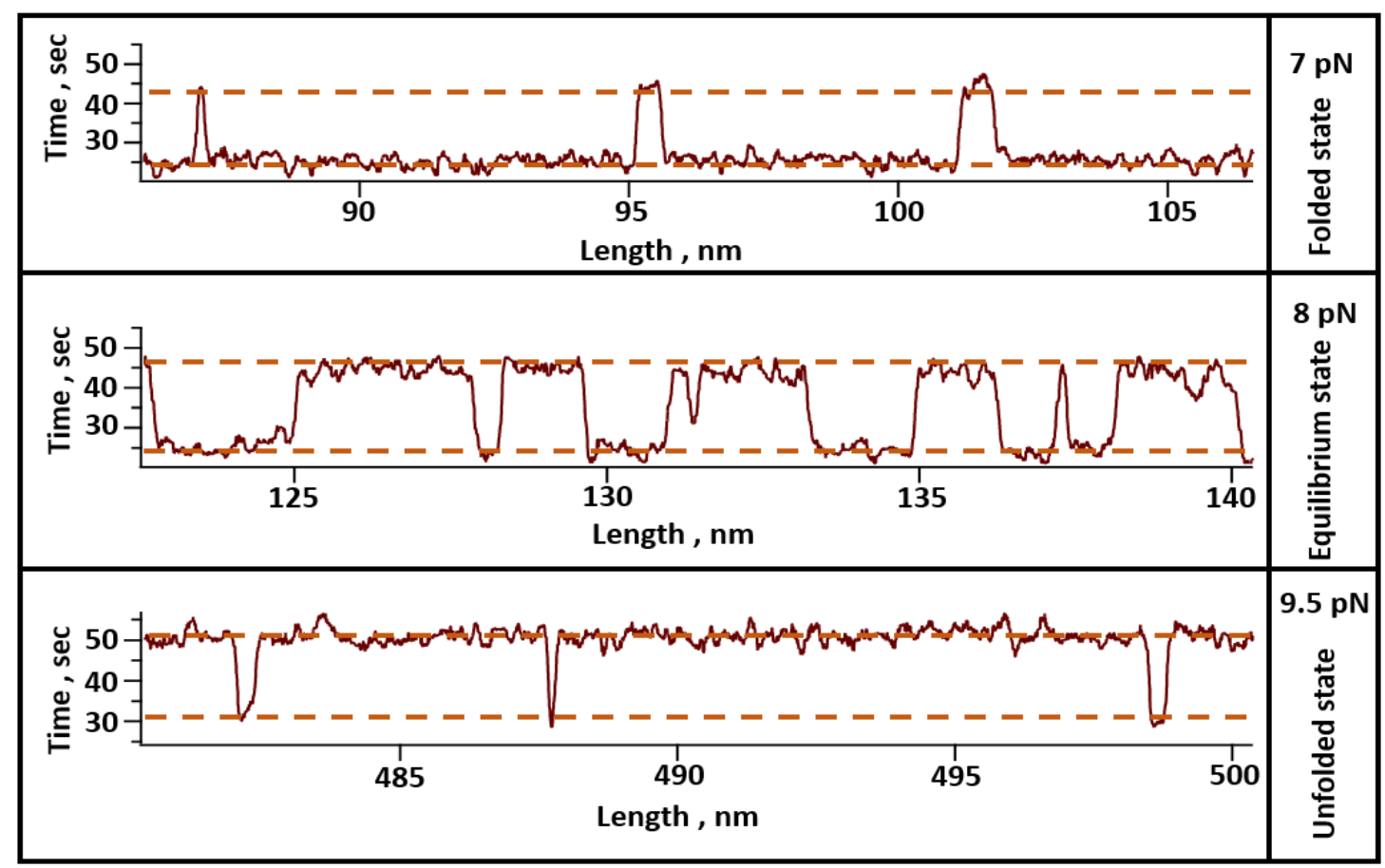


Supplementary figure 12: Representative trace of talin in presence of $1 \mu M$ DnaJ: In presence of $1 \mu M$ DnaJ, folding dynamics of talin has been shifted towards lower force regime. At $7 p N$ force talin mostly in the folded state (top trace) and at $9 p N$ force it mostly in the unfolded state. At $8 \mathrm{pN}$, folding probability is almost 0.5 where it stays almost $50 \%$ time in the folded state.

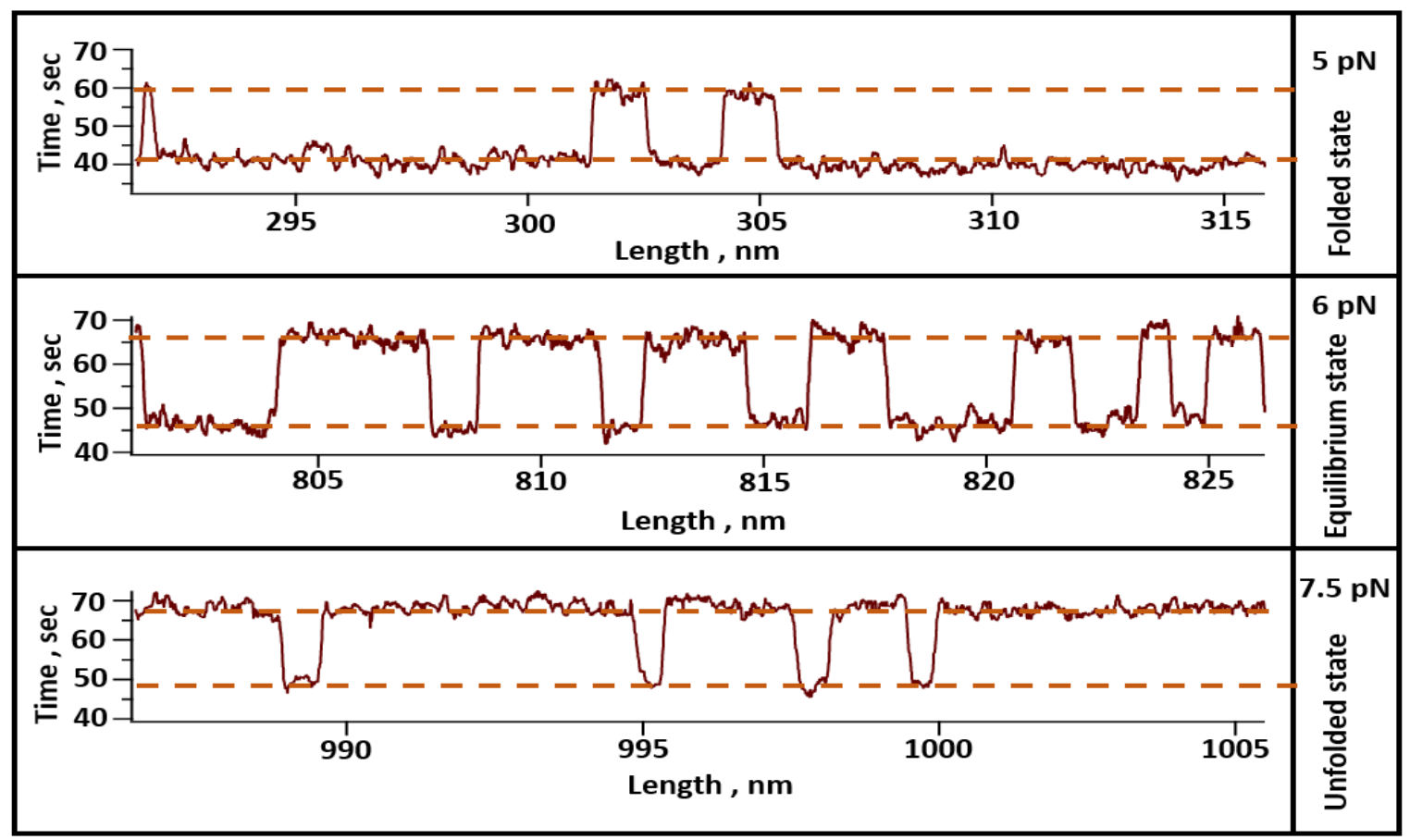

Supplementary figure 13: Representative trace of talin in presence of $3 \mu M$ DnaK: In presence of $3 \mu M$ DnaK, folding dynamics of talin has been shifted towards lower force regime and mostly in the folded state at $5 \mathrm{pN}$ (top trace). Whereas at $6 \mathrm{pN}$ both folded and unfolded state are almost equally populated. At $7.5 \mathrm{pN}$ it mostly populated in the unfolded state (bottom trace). 


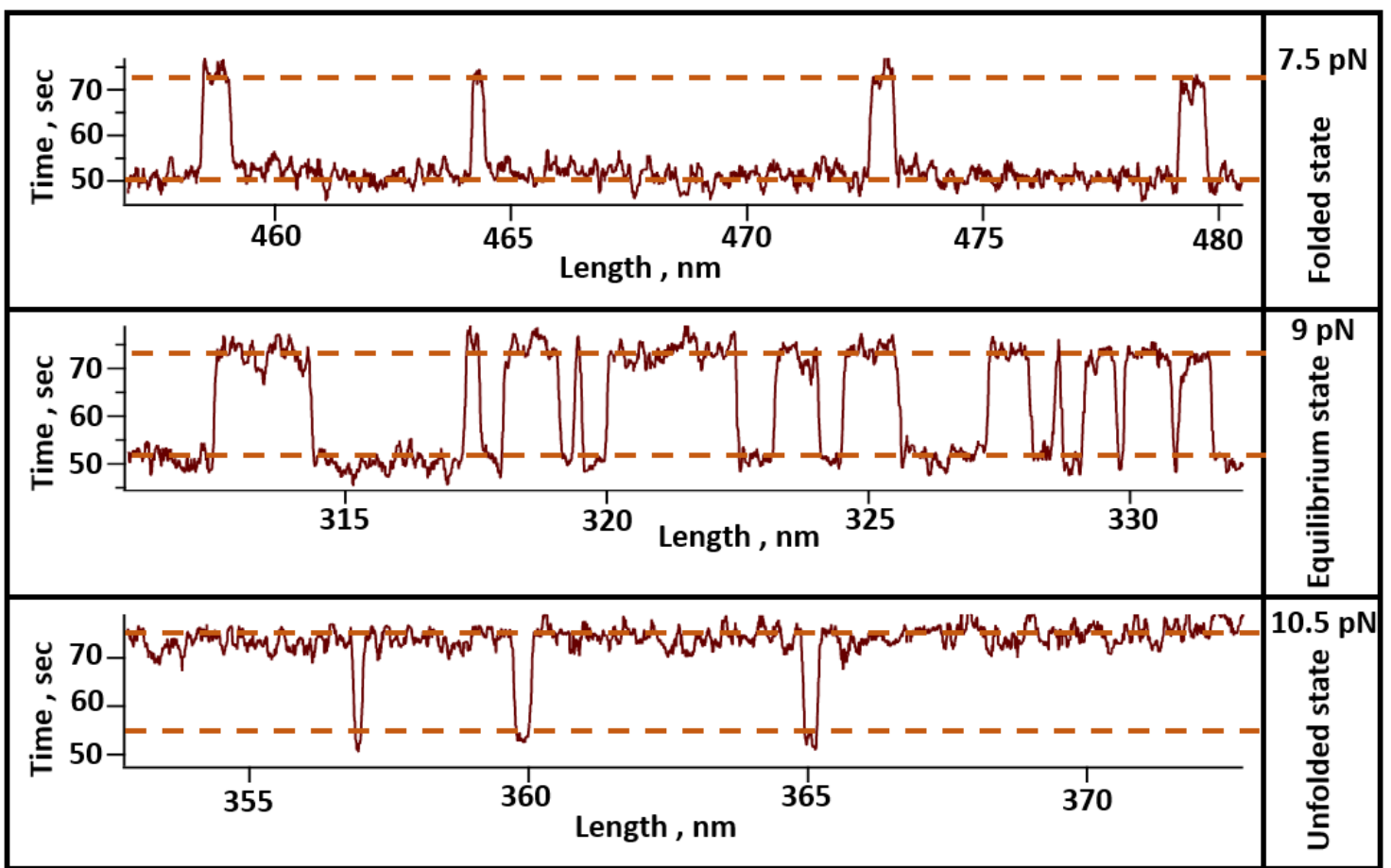

Supplementary figure 14: Representative trace of talin in presence of DnaKJ-ATP complex: Here the dynamics of talin with force been monitored in the presence of $1 \mu M$ DnaJ, $3 \mu \mathrm{M}$ DnaK and $10 \mathrm{mM}$ ATP and $10 \mathrm{mM} \mathrm{MgCl}$. The buffer is exchanged after every 30 minutes with fresh ATP to keep the sufficient supply of ATP. In this condition, talin mostly in the folded state (top trace) at $7.5 \mathrm{pN}$ and at $9 \mathrm{pN}$, both unfolded and folded states are equally populated. At $10.5 \mathrm{pN}$ it mostly in the unfolded state (bottom trace). 


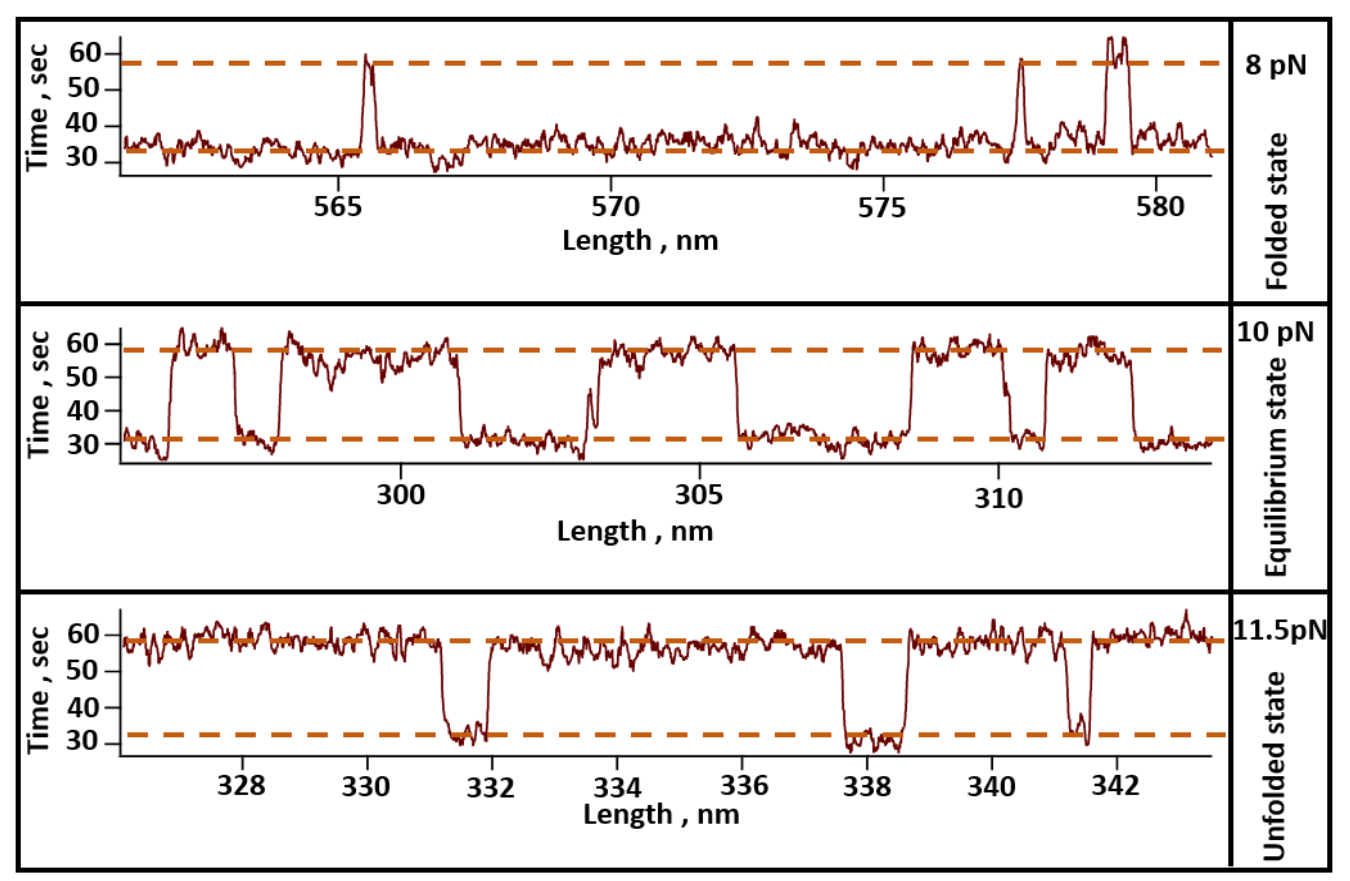

Supplementary figure 15: Representative trace of talin in presence of DnaKJE-ATP conplex: Here the dynamics of talin has been monitored in the presence of $1 \mu M$ DnaJ, $3 \mu M$ DnaK, $5 \mu M$ GrpE and $10 \mathrm{mM} \mathrm{ATP}$ and $10 \mathrm{mM} \mathrm{MgCl}$. The buffer is exchanged after every 30 minutes with fresh ATP to keep the sufficient supply of ATP. DnaJKE complex restores the ability of folding dynamics of talin and it almost in the folded state (top trace) at $8 \mathrm{pN}$ and totally unfolded at $11.5 \mathrm{pN}$ (bottom trace). 


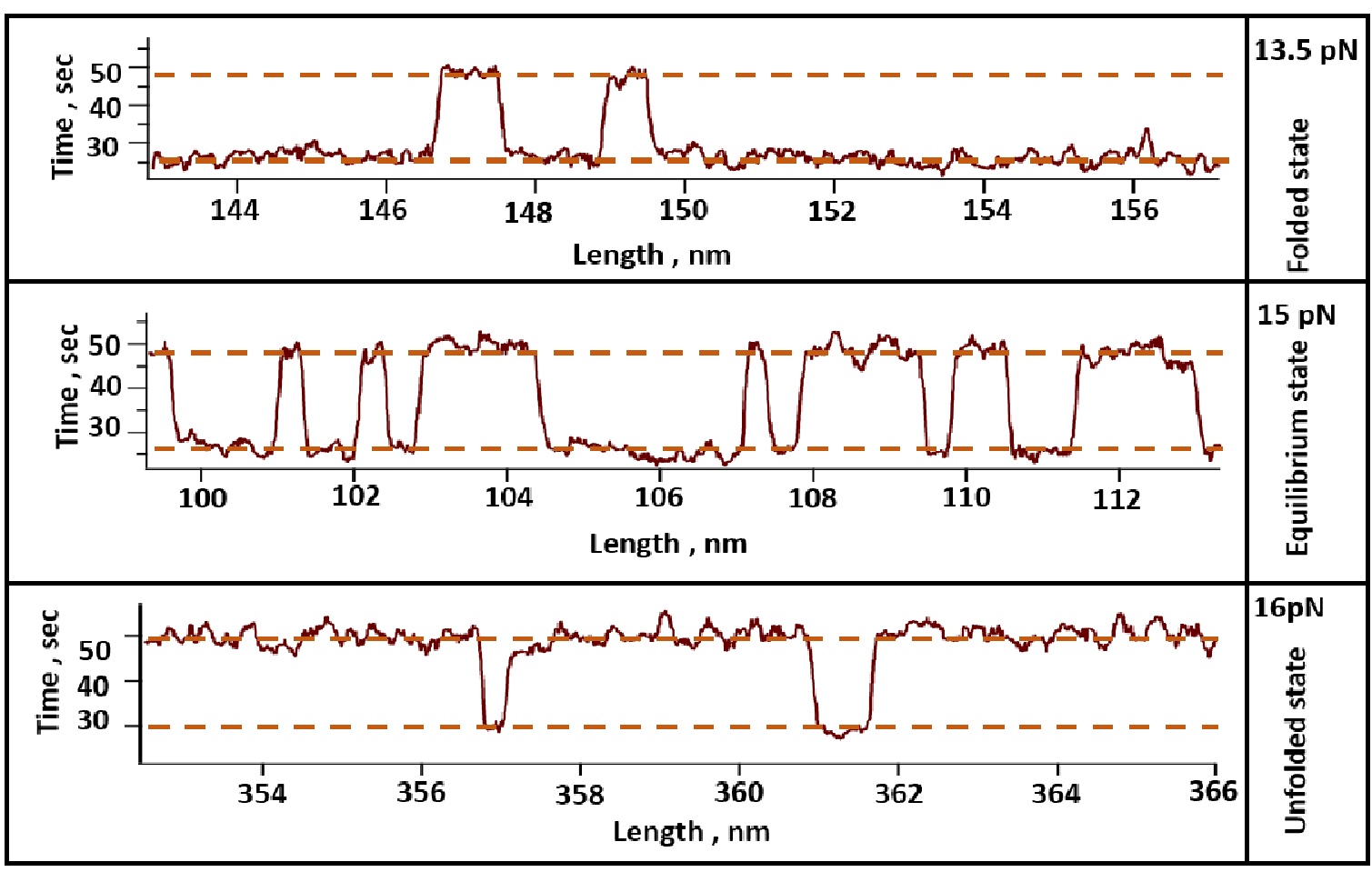

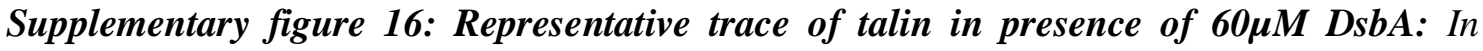
presence of $60 \mu M$ DsbA, folding dynamics of talin has been shifted towards higher force regime and mostly in the folded state (top trace) at $13.5 \mathrm{pN}$. Whereas at $15 \mathrm{pN}$ both folded and unfolded state are equally populated. At $16 \mathrm{pN}$ force it mostly populated in the unfolded state (bottom trace). 